\title{
Regularised Finite Difference Methods for the Logarithmic Klein-Gordon Equation
}

\author{
Jingye Yan $^{1}$, Hong Zhang ${ }^{1}$, Xu Qian ${ }^{1, *}$ and Songhe Song ${ }^{1,2}$ \\ ${ }^{1}$ Department of Mathematics, College of Arts and Sciences, National University of \\ Defense Technology, Changsha 410073, China. \\ ${ }^{2}$ State Key Laboratory of High Performance Computing, National University of \\ Defense Technology, Changsha 410073, China.
}

Received 14 August 2020; Accepted (in revised version) 25 August 2020.

\begin{abstract}
Two regularised finite difference methods for the logarithmic Klein-Gordon equation are studied. In order to deal with the origin singularity, we employ regularised logarithmic Klein-Gordon equations with a regularisation parameter $0<\varepsilon \ll 1$. Two finite difference methods are applied to the regularised equations. It is proven that the methods have the second order of accuracy both in space and time. Numerical experiments show that the solutions of the regularised equations converge to the solution of the initial equation as $\mathscr{O}(\varepsilon)$.
\end{abstract}

AMS subject classifications: 35Q40, 65M15, 81Q05

Key words: Logarithmic Klein-Gordon equation, regularised logarithmic Klein-Gordon equation, finite difference method, error estimate, convergence order.

\section{Introduction}

The logarithmic Klein-Gordon equation (LogKGE), also known as the relativistic version of the logarithmic Schrödinger equation [15], has been introduced in the quantum field theory by Rosen [41]. It has the form

$$
\begin{aligned}
& u_{t t}(\mathbf{x}, t)-\Delta u(\mathbf{x}, t)+u(\mathbf{x}, t)+\lambda u(\mathbf{x}, t) \ln \left(|u(\mathbf{x}, t)|^{2}\right)=0, \quad \mathbf{x} \in \mathbb{R}^{d}, \quad t>0, \\
& u(\mathbf{x}, 0)=\phi(\mathbf{x}), \quad \partial_{t} u(\mathbf{x}, 0)=\gamma(\mathbf{x}), \quad \mathbf{x} \in \mathbb{R}^{d},
\end{aligned}
$$

where $\mathbf{x}=\left(x_{1}, \ldots, x_{d}\right)^{\mathrm{T}} \in \mathbb{R}^{d}, d=1,2,3$ is the spatial coordinate, $t$ time, $u:=u(\mathbf{x}, t)$ a realvalued scalar field, and $\lambda$ shows the force of the non-linear interaction. Such non-linearities appear in relativistic wave equations, which describe dilatonic quantum gravity [43], superfluid [44], spinless particles [16,17] and non-relativistic spinning particles moving in an external electromagnetic field. Besides, such non-linearity effects often arise in various

${ }^{*}$ Corresponding author. Email addresses: yanjingye0205@163.com (J. Yan), zhanghnudt@163.com (H. Zhang), qianxu@nudt.edu.cn (X. Qian), shsong@nudt.edu.cn (S. Song) 
areas of physics such as inflation cosmology $[14,25]$, supersymmetric field theories, geophysics [32,37], optics [20], and nuclear physics [34]. The LogKGE (1.1) has been also used to describe spinless particles in optics [42]. If $u(\cdot, t) \in H^{1}\left(\mathbb{R}^{d}\right)$ and $\partial_{t} u(\cdot, t) \in L^{2}\left(\mathbb{R}^{d}\right)$, then the LogKGE (1.1) admits the energy conservation law - cf. [35,38], i.e.

$$
\begin{aligned}
E(t) & =\int_{\Omega}\left[\left|u_{t}(\mathbf{x}, t)\right|^{2}+|\nabla u(\mathbf{x}, t)|^{2}+(1-\lambda)|u(\mathbf{x}, t)|^{2}+\lambda|u(\mathbf{x}, t)|^{2} \ln \left(|u(\mathbf{x}, t)|^{2}\right)\right] \mathrm{d} x \\
& \equiv E(0) .
\end{aligned}
$$

The global-in-time well-posedness of the solution to the Klein-Gordon equation with a logarithmic potentials attracted considerable attention. Thus Cazenave and Haraux [22] studied the local existence and uniqueness of solution of the Cauchy problem. Later on, Górka [27] used the compactness method to show the global existence of weak solutions for one-dimensional equations in bounded domains. Bartkowski and Górka [15] studied the corresponding Cauchy problem on the real line $\mathbb{R}$ without boundary conditions. They established the global existence of weak solutions, classical solutions and traveling waves. Natali and Cardoso Jr. [39] used compactness arguments and a non-standard analysis to prove the existence and uniqueness of weak solutions for an associated Cauchy problem in the energy space. Bialynicki-Birula and Mycielski [18] studied the Gaussons - i.e. the solutions, which represent the Gaussian shape [48]. Note that the interaction of Gaussons was first considered by Makhankov et al. [36]. For the nonlinear Klein-Gordon equation (NKGE) and the oscillatory NKGE, Cauchy problems, well-posedness and dynamical properties are investigated in $[1,19,29,31,45]$.

Numerical methods have for the non-linear Klein-Gordon equation (NKGE) and the oscillatory NKGE have been also developed. Thus standard finite difference time domain (FDTD) methods such as energy conservative, semi-implicit, explicit finite difference time domain are considered in $[8,10,23,24,47,50]$, a multiscale time integrator Fourier pseudospectral (MTIFP) method in [4,11,12], a finite element method in [21], an exponential wave integrator Fourier pseudospectral (EWI-FP) method in [8,9], and an asymptotic preserving (AP) method in [26]. For numerical comparison of various numerical methods for the NKGE and the oscillatory NKGE, we refer the reader to $[8,13,30,40]$. Stochastic conformal Preissman, stochastic conformal discrete gradient and stochastic conformal Euler box schemes for damped stochastic Klein-Gordon equation are presented in [46]. Nevertheless, these methods can not be directly applied to the LogKGE equation (1.1) because of the singularity of the logarithmic non-linearity at the origin.

Considering the logarithmic Schrödinger equation (LogSE), Bao et al. [6] employed regularised mass and energy conservative finite difference methods in order to avoid the blowup of the logarithmic non-linearity. Latter on, a regularised semi-implicit difference scheme for the LogSE was studied in [5]. Li et al. [33] proposed a Crank-Nicolson-type finite difference method for the LogSE on unbounded domains. Zhang et al. [49] developed high order diagonally Runge-Kutta schemes for the LogSE, which preserve the mass and quadratic energy and introduced multi-symplectic integrators which conserve mass. In order to avoid the singularity of the logarithmic function, Bao et al. [7] applied Lie-Trotter 
splitting integrators in energy regularization for the LogSE. Using the ideas of $[5,6,49]$, we consider a regularised logarithmic Klein-Gordon equation (RLogKGE) with a small regularised parameter $0<\varepsilon \ll 1$, viz.

$$
\begin{aligned}
& u_{t t}^{\varepsilon}(\mathbf{x}, t)-\Delta u^{\varepsilon}(\mathbf{x}, t)+u^{\varepsilon}(\mathbf{x}, t)+\lambda u^{\varepsilon}(\mathbf{x}, t) \ln \left(\varepsilon^{2}+\left|u^{\varepsilon}(\mathbf{x}, t)\right|^{2}\right)=0, \quad \mathbf{x} \in \mathbb{R}^{d}, \quad t>0, \\
& u^{\varepsilon}(\mathbf{x}, 0)=\phi(\mathbf{x}), \quad \partial_{t} u^{\varepsilon}(\mathbf{x}, 0)=\gamma(\mathbf{x}), \quad \mathbf{x} \in \mathbb{R}^{d} .
\end{aligned}
$$

This equation is time symmetric or time reversible - i.e. it preserves its form when $t$ is replaced by $-t$.

Remark 1.1. The Cauchy problems for the Eqs. (1.1) and (1.2), the convergence estimate between the regularised model (1.2) and the LogKGE (1.1) will be considered elsewhere.

Theorem 1.1. If $u^{\varepsilon}(\cdot, t) \in H^{1}\left(\mathbb{R}^{d}\right)$ and $\partial_{t} u^{\varepsilon}(\cdot, t) \in L^{2}\left(\mathbb{R}^{d}\right)$, then the RLogKGE (1.2) follows the energy conservation law - i.e.

$$
E^{\varepsilon}(t)=\int_{\Omega}\left[\left|u_{t}^{\varepsilon}(\mathbf{x}, t)\right|^{2}+\left|\nabla u^{\varepsilon}(\mathbf{x}, t)\right|^{2}+\left|u^{\varepsilon}(\mathbf{x}, t)\right|^{2}+\lambda F_{\varepsilon}\left(\left|u^{\varepsilon}(\mathbf{x}, t)\right|^{2}\right)\right] \mathrm{d} \mathbf{x} \equiv E^{\varepsilon}(0),
$$

where

$$
F_{\varepsilon}(\rho)=\int_{0}^{\rho} \ln \left(\varepsilon^{2}+s\right) \mathrm{d} s=\rho \ln \left(\varepsilon^{2}+\rho\right)+\varepsilon^{2} \ln \left(1+\frac{\rho}{\varepsilon^{2}}\right)-\rho, \quad \rho=\left|u^{\varepsilon}(\mathbf{x}, t)\right|^{2} .
$$

Proof. Computing the derivative

$$
\begin{aligned}
\frac{d}{d t} E^{\varepsilon}(t) & =2 \int_{\Omega}\left[u_{t}^{\varepsilon} \cdot u_{t t}^{\varepsilon}+\nabla u^{\varepsilon} \cdot \nabla u_{t}^{\varepsilon}+u^{\varepsilon} u_{t}^{\varepsilon}+\lambda F_{\varepsilon}^{\prime}\left(\left|u^{\varepsilon}\right|^{2}\right) \cdot u^{\varepsilon} \cdot u_{t}^{\varepsilon}\right](\mathbf{x}, t) \mathrm{d} \mathbf{x} \\
& =2 \int_{\Omega}\left[u_{t}^{\varepsilon}\left(u_{t t}^{\varepsilon}-\Delta u^{\varepsilon}+u^{\varepsilon}+\lambda u^{\varepsilon} \ln \left(\varepsilon^{2}+\left|u^{\varepsilon}\right|^{2}\right)\right)\right](\mathbf{x}, t) \mathrm{d} \mathbf{x}=0
\end{aligned}
$$

leads to the result stated.

The aim of this work is to study the efficiency of the Eqs. (1.1) and (1.2) and to investigate two FDTD schemes for (1.2).

The rest of this paper is organised as follows. In Section 2, a semi-implicit and an explicit FDTD schemes for the RLogKGE (1.2) are proposed and they stability and solvability are studied. Errors of the methods are discussed in Section 3. In Section 4 we carry out numerical experiments to verify the theoretical results. Some concluding remarks are drawn in Section 5.

\section{FDTD Methods and Their Stability}

In this section, we construct two FDTD schemes for the Eq. (1.2), study their stability, solvability and error estimates. For simplicity, we set $\lambda=1$ and construct and analyse 
numerical schemes in the one dimensional space only. Thus we consider the Eq. (1.2) with periodic boundary conditions - i.e.

$$
\begin{aligned}
& \partial_{t t} u^{\varepsilon}(x, t)-\partial_{x x} u^{\varepsilon}(x, t)+u^{\varepsilon}(x, t) \\
& \quad+u^{\varepsilon}(x, t) \ln \left(\varepsilon^{2}+\left|u^{\varepsilon}(x, t)\right|^{2}\right)=0, \quad x \in \Omega=(a, b), \quad t>0, \\
& u^{\varepsilon}(x, 0)=\phi(x), \quad \partial_{t} u^{\varepsilon}(x, 0)=\gamma(x), \quad x \in \bar{\Omega}=[a, b] .
\end{aligned}
$$

Choose a time step $\tau:=\Delta t$ and set $t_{n}:=n \tau, n=0,1,2, \ldots$ Besides, let $N$ be a positive integer,

$$
\mathscr{T}_{N}:=\{j \mid j=0,1,2, \ldots, N-1\}, \quad \mathscr{T}_{N}^{0}:=\{j \mid j=0,1,2, \ldots, N\},
$$

the index sets, $h:=(b-a) / N$ and $x_{j}:=a+j h, j=0,1, \ldots, N$. Assuming that $u_{j}^{\varepsilon, n}$ and $u_{j}^{n}, j \in \mathscr{T}_{N}^{0}, n \geq 0$ are, respectively, approximations of the exact solutions $u^{\varepsilon}\left(x_{j}, t_{n}\right)$ and $u\left(x_{j}, t_{n}\right)$, we introduce the numerical solution vectors

$$
u^{\varepsilon, n}=\left(u_{0}^{\varepsilon, n}, u_{1}^{\varepsilon, n}, \ldots, u_{N}^{\varepsilon, n}\right)^{\mathrm{T}}, \quad u^{n}=\left(u_{0}^{n}, u_{1}^{n}, \ldots, u_{N}^{n}\right)^{\mathrm{T}} \in \mathbb{R}^{N+1}
$$

at time $t=t_{n}$. The finite difference operators are defined as follows:

$$
\begin{array}{ll}
\delta_{t}^{+} u_{j}^{n}=\frac{u_{j}^{n+1}-u_{j}^{n}}{\tau}, \quad \delta_{t}^{-} u_{j}^{n}=\frac{u_{j}^{n}-u_{j}^{n-1}}{\tau}, \quad \delta_{t}^{2} u_{j}^{n}=\frac{u_{j}^{n+1}-2 u_{j}^{n}+u_{j}^{n-1}}{\tau^{2}}, \\
\delta_{x}^{+} u_{j}^{n}=\frac{u_{j+1}^{n}-u_{j}^{n}}{h}, \quad \delta_{x}^{-} u_{j}^{n}=\frac{u_{j}^{n}-u_{j-1}^{n}}{h}, \quad \delta_{x}^{2} u_{j}^{n}=\frac{u_{j+1}^{n}-2 u_{j}^{n}+u_{j-1}^{n}}{h^{2}} .
\end{array}
$$

We also consider the space of grid functions

$$
X_{N}:=\left\{u \mid u=\left(u_{0}, u_{1}, u_{2}, \ldots, u_{N}\right)^{\mathrm{T}}, u_{0}=u_{N}\right\} \subseteq \mathbb{R}^{N+1}
$$

and use standard discrete $l^{2}$, semi- $H^{1}$ and $l^{\infty}$ norms and inner product for $X_{N}$, viz.

$$
\begin{array}{ll}
\|u\|_{l^{2}}^{2}=(u, u)=h \sum_{j=0}^{N-1}\left|u_{j}\right|^{2}, & \left\|\delta_{x}^{+} u\right\|_{l^{2}}^{2}=h \sum_{j=0}^{N-1}\left|\delta_{x}^{+} u_{j}\right|^{2}, \\
\|u\|_{l^{\infty}}=\sup _{0 \leq j \leq N-1}\left|u_{j}\right|, & (u, v)=h \sum_{j=0}^{N-1} u_{j} v_{j},
\end{array}
$$

where $u, v \in X_{N}$, and

$$
\left(\delta_{x}^{2} u, v\right)=-\left(\delta_{x}^{+} u, \delta_{x}^{+} v\right)=\left(u, \delta_{x}^{2} v\right) .
$$

For the numerical solution of the Eq. (1.2), we employ the following FDTD methods.

I. Semi-implicit finite difference (SIFD) scheme.

$$
\begin{aligned}
\delta_{t}^{2} u_{j}^{\varepsilon, n} & -\frac{1}{2} \delta_{x}^{2}\left(u_{j}^{\varepsilon, n+1}+u_{j}^{\varepsilon, n-1}\right)+\frac{1}{2}\left(u_{j}^{\varepsilon, n+1}+u_{j}^{\varepsilon, n-1}\right) \\
& +u_{j}^{\varepsilon, n} f_{\varepsilon}\left(\left|u_{j}^{\varepsilon, n}\right|^{2}\right)=0, \quad n \geq 1
\end{aligned}
$$


II. Explicit finite difference (EXFD) scheme.

$$
\delta_{t}^{2} u_{j}^{\varepsilon, n}-\delta_{x}^{2} u_{j}^{\varepsilon, n}+u_{j}^{\varepsilon, n}+u_{j}^{\varepsilon, n} f_{\varepsilon}\left(\left|u_{j}^{\varepsilon, n}\right|^{2}\right)=0, \quad n \geq 1,
$$

where $f_{\varepsilon}(\rho)=\ln \left(\varepsilon^{2}+\rho\right)$.

The initial and boundary conditions are discretised as follows:

$$
u_{0}^{\varepsilon, n+1}=u_{N}^{\varepsilon, n+1}, \quad u_{-1}^{\varepsilon, n+1}=u_{N-1}^{\varepsilon, n+1}, \quad n \geq 0, \quad u_{j}^{\varepsilon, 0}=\phi\left(x_{j}\right), \quad j \in \mathscr{T}_{N}^{0} .
$$

Using the Taylor expansion, we get a first step solution $u_{j}^{\varepsilon, 1}$, viz.

$$
u_{j}^{\varepsilon, 1}=\phi\left(x_{j}\right)+\tau \gamma\left(x_{j}\right)+\frac{\tau^{2}}{2}\left[\delta_{x}^{2} \phi\left(x_{j}\right)-\phi\left(x_{j}\right)-\phi\left(x_{j}\right) \ln \left(\varepsilon^{2}+\left|\phi\left(x_{j}\right)\right|^{2}\right)\right], \quad j \in \mathscr{T}_{N}^{0} .
$$

It is easily seen that these FDTD schemes are all time symmetric, i.e. they do not change their form if $n+1$ is replaced by $n-1$ and $\tau$ by $-\tau$.

We now consider the stability of the methods. Let $T_{\max }$ be the maximum existence time and $0<T<T_{\max }$. Define

$$
\sigma_{\max }:=\max \left\{\left|\ln \left(\varepsilon^{2}\right)\right|,\left|\ln \left(\varepsilon^{2}+\left\|u^{\varepsilon, n}\right\|_{l^{\infty}}^{2}\right)\right|\right\}, \quad 0 \leq n \leq \frac{T}{\tau}-1 .
$$

Theorem 2.1. Consider the FDTD schemes (2.2) and (2.3) till $t=T$.

(i) If $0 \leq \sigma_{\max } \leq 1$, then the SIFD scheme (2.2) is unconditionally stable.

(ii) If $\sigma_{\max }>1$, then the SIFD scheme (2.2) is conditionally stable for all $\tau$ such that

$$
\tau \leq \frac{2}{\sqrt{\sigma_{\max }-1}} .
$$

(iii) The EXFD scheme (2.3) is conditionally stable for all $\tau$ such that

$$
\tau \leq \frac{2 h}{\sqrt{\left(\sigma_{\max }+1\right) h^{2}+4}} .
$$

Proof. Let $\xi_{l}$ refer to the amplification factor of the $l$-th mode in the phase space. Substituting

$$
u_{j}^{\varepsilon, n-1}=\sum_{l} \hat{U}_{l} e^{2 i j l \pi / N}, \quad u_{j}^{\varepsilon, n}=\sum_{l} \xi_{l} \hat{U}_{l} e^{2 i j l \pi / N}, \quad u_{j}^{\varepsilon, n+1}=\sum_{l} \xi_{l}^{2} \hat{U}_{l} e^{2 i j l \pi / N}
$$

into the Eqs. (2.2),(2.3) leads to the characteristic equation

$$
\xi_{l}^{2}-2 \theta_{l} \xi_{l}+1=0, \quad l=-\frac{N}{2}, \ldots, \frac{N}{2}-1,
$$


where $\theta_{l}$ is an invariant for different methods. It follows that $\xi_{l}=\theta_{l} \pm \sqrt{\theta_{l}^{2}-1}$. The stability of the numerical schemes leads to the condition $\left|\xi_{l}\right| \leq 1$, which is equivalent to

$$
\left|\theta_{l}\right| \leq 1, \quad l=-\frac{N}{2}, \ldots, \frac{N}{2}-1 .
$$

Setting

$$
s_{l}=\frac{2}{h} \sin \left(\frac{l \pi}{N}\right), \quad l=-\frac{N}{2}, \ldots, \frac{N}{2}-1,
$$

we have

$$
0 \leq s_{l}^{2} \leq \frac{4}{h^{2}}
$$

First, we establish the linear stability. Assuming that $f_{\varepsilon}\left(\left|u^{\varepsilon}\right|^{2}\right)=\alpha$ for a constant $\alpha>0$ implies the linearity of (2.2) and (2.3).

(i) For the SIFD scheme (2.2), we have

$$
\theta_{l}=\frac{2-\alpha \tau^{2}}{2+\tau^{2}\left(s_{l}^{2}+1\right)}, \quad l=-\frac{N}{2}, \ldots, \frac{N}{2}-1
$$

If $0 \leq \alpha \leq 1$, then $\left|\theta_{l}\right| \leq 1$ and the SIFD scheme (2.2) is unconditionally stable. On the other hand, if $\alpha>1$, then

$$
2-\alpha \tau^{2} \geq-2-\tau^{2}
$$

This implies the stability of the SIFD scheme (2.2) for $\tau \leq 2 / \sqrt{\alpha-1}$.

(ii) For the EXFD scheme (2.3), we have

$$
\theta_{l}=\frac{2-\tau^{2}\left(1+\alpha+s_{l}^{2}\right)}{2}, \quad l=-\frac{N}{2}, \ldots, \frac{N}{2}-1 .
$$

It follows from (2.8) that

$$
\tau^{2}\left(\alpha+1+s_{l}^{2}\right) \leq \tau^{2}\left(\alpha+1+\frac{4}{h^{2}}\right) \leq 4 \Rightarrow\left|\theta_{l}\right|<1 .
$$

Therefore, if

$$
\tau \leq \frac{2 h}{\sqrt{(\alpha+1) h^{2}+4}}
$$

the EXFD scheme (2.3) is stable.

Consider now non-linear schemes. The same approach shows that if

$$
\tau \leq \frac{2}{\sqrt{\sigma_{\max }-1}}
$$


then the nonlinear SIFD scheme is stable. Besides, if

$$
\tau \leq \frac{2 h}{\sqrt{\left(\sigma_{\max }+1\right) h^{2}+4}}
$$

then the non-linear EXFD scheme (2.3) is stable.

Remark 2.1. Since the scheme SIFD (2.2) is linear, and the coefficient matrix is strictly diagonal, it is easy to conclude that the SIFD (2.2) is solvable. In addition, EXFD (2.3) is explicit, so it is clear that there is a unique solution.

\section{Error Estimates}

Motivated by analytical results concerning the FDTD schemes for the Klein-Gordon equation [8,10], for logarithmic Schrödinger equation [5], and for Schrödinger and GrossPitaevskii equations [2,3], we derive error estimates for the methods mentioned.

Condition 3.1. The solution $u^{\varepsilon}$ of the Eq. (1.2) on $\Omega_{T}: \Omega \times[0, T]$ belongs to the set

$$
C\left([0, T] ; H^{5}(\Omega)\right) \cap C^{2}\left([0, T] ; H^{4}(\Omega)\right) \cap C^{4}\left([0, T] ; H^{2}(\Omega)\right),
$$

and there exist $\varepsilon_{0}>0$ and $C_{0}>0$ independent of $\varepsilon$ such that the inequality

$$
\begin{aligned}
& \left\|u^{\varepsilon}\right\|_{L^{\infty}\left(0, T ; H^{5}(\Omega)\right)}+\left\|\partial_{t}^{2} u^{\varepsilon}\right\|_{L^{\infty}\left(0, T ; H^{4}(\Omega)\right)} \\
& \quad+\left\|\partial_{t}^{3} u^{\varepsilon}\right\|_{L^{\infty}\left(0, T ; H^{2}(\Omega)\right)}+\left\|\partial_{t}^{4} u^{\varepsilon}\right\|_{L^{\infty}\left(0, T ; H^{2}(\Omega)\right)} \leq C_{0}
\end{aligned}
$$

holds uniformly in $0 \leq \varepsilon \leq \varepsilon_{0}$. by

Set $\Lambda:=\left\|u^{\varepsilon}(x, t)\right\|_{L^{\infty}\left(\Omega_{T}\right)}$ and consider the grid error function $e^{\varepsilon, n} \in X_{N}(n \geq 0)$ defined

$$
e_{j}^{\varepsilon, n}=u^{\varepsilon}\left(x_{j}, t_{n}\right)-u_{j}^{\varepsilon, n}, \quad j \in \mathscr{T}_{N}^{0}, \quad n=0,1,2, \ldots,
$$

where $u^{\varepsilon}$ and $u_{j}^{\varepsilon, n}$ are the exact solution and numerical approximation of (2.1), respectively.

Theorem 3.1. If Condition 3.1 holds, then there exist $h_{0}>0, \tau_{0}>0$ sufficiently small with $h_{0}^{2} \sim e^{-(T / 2)\left(\ln \left(\varepsilon^{2}\right)\right)^{2}}, \tau_{0}^{2} \sim e^{-(T / 2)\left(\ln \left(\varepsilon^{2}\right)\right)^{2}}$, for any $0<\varepsilon \ll 1$, when $0<h \leq h_{0}$ and $0<\tau \leq$ $\tau_{0}$ and under the stability condition (2.6), the SIFD (2.2) with (2.4) and (2.5) satisfies the following error estimates

$$
\left\|\delta_{x}^{+} e^{\varepsilon, n}\right\|_{l^{2}}+\left\|e^{\varepsilon, n}\right\|_{l^{2}} \lesssim e^{(T / 2)\left(\ln \left(\varepsilon^{2}\right)\right)^{2}}\left(\tau^{2}+h^{2}\right), \quad\left\|u^{\varepsilon, n}\right\|_{l^{\infty}} \leq \Lambda+1 .
$$

Note that here and in what follows, the notation $p \lesssim q$ means that there is a constant $C$ independent of $\tau, h, \varepsilon$ such that $|p| \leq C q$.

Theorem 3.2. Under Condition 3.1 and $\tau \leq(1 / 2) \min \{1, h\}$, there exist $h_{0}>0, \tau_{0}>0$ sufficiently small with $h_{0}^{2} \sim e^{-(T / 2)\left(\ln \left(\varepsilon^{2}\right)\right)^{2}}, \tau_{0}^{2} \sim e^{-(T / 2)\left(\ln \left(\varepsilon^{2}\right)\right)^{2}}$, for any $0<\varepsilon \ll 1$, when $0<h \leq h_{0}$ and $0<\tau \leq \tau_{0}$ and under the stability condition (2.7), the EXFD (2.3) with (2.4) and (2.5) satisfies the error estimates

$$
\left\|\delta_{x}^{+} e^{\varepsilon, n}\right\|_{l^{2}}+\left\|e^{\varepsilon, n}\right\|_{l^{2}} \lesssim e^{(T / 2)\left(\ln \left(\varepsilon^{2}\right)\right)^{2}}\left(\tau^{2}+h^{2}\right), \quad\left\|u^{\varepsilon, n}\right\|_{l^{\infty}} \leq \Lambda+1 .
$$


Remark 3.1 (cf. Bao at al. [8,10]). For two- and three-dimensional equations, the above theorems are valid under the conditions $0<h^{2} \lesssim e^{-(T / 2)\left(\ln \left(\varepsilon^{2}\right)\right)^{2}} C_{d}(h)$ and $0<\tau^{2} \lesssim$ $e^{-(T / 2)\left(\ln \left(\varepsilon^{2}\right)\right)^{2}} C_{d}(h)$. Besides, the inverse inequality takes the form

$$
\left\|u^{\varepsilon, n}\right\|_{l^{\infty}} \lesssim \frac{1}{C_{d}(h)}\left(\left\|\delta_{x}^{+} u^{\varepsilon, n}\right\|_{l^{2}}+\left\|u^{\varepsilon, n}\right\|_{l^{2}}\right)
$$

where $C_{d}(h)=1 /|\ln h|$ for $d=2$ and $C_{d}(h)=h^{1 / 2}$ for $d=3$.

The rest of this section is devoted to the proof of Theorems 3.1 and 3.2.

\subsection{Proof of Theorem 3.1 for the SIFD}

Defining local truncation errors for the SIFD (2.2) as

$$
\begin{aligned}
\xi_{j}^{\varepsilon, 0}:= & \delta_{t}^{+} u^{\varepsilon}\left(x_{j}, 0\right)-\gamma\left(x_{j}\right)-\frac{\tau}{2}\left[\delta_{x}^{2} \phi\left(x_{j}\right)-\phi\left(x_{j}\right)-\phi\left(x_{j}\right) \ln \left(\varepsilon^{2}+\left|\phi\left(x_{j}\right)\right|^{2}\right)\right], \\
\xi_{j}^{\varepsilon, n}:= & \delta_{t}^{2} u^{\varepsilon}\left(x_{j}, t_{n}\right)-\frac{1}{2} \delta_{x}^{2}\left(u^{\varepsilon}\left(x_{j}, t_{n+1}\right)+u^{\varepsilon}\left(x_{j}, t_{n-1}\right)\right) \\
& +\frac{1}{2}\left(u^{\varepsilon}\left(x_{j}, t_{n+1}\right)+u^{\varepsilon}\left(x_{j}, t_{n-1}\right)\right) \\
& +u^{\varepsilon}\left(x_{j}, t_{n}\right) f_{\varepsilon}\left(\left(u^{\varepsilon}\left(x_{j}, t_{n}\right)\right)^{2}\right), \quad j \in \mathscr{T}_{N}, \quad 1 \leq n \leq \frac{T}{\tau}-1,
\end{aligned}
$$

we can estimate them as follows.

Lemma 3.1. If Condition 3.1 holds, then

$$
\begin{gathered}
\left\|\xi^{\varepsilon, 0}\right\|_{H^{1}} \lesssim h^{2}+\tau^{2}, \quad\left\|\xi^{\varepsilon, n}\right\|_{l^{2}} \lesssim h^{2}+\tau^{2}, \\
\left\|\delta_{x}^{+} \xi^{\varepsilon, n}\right\|_{l^{2}} \lesssim h^{2}+\tau^{2}, \quad 1 \leq n \leq \frac{T}{\tau}-1 .
\end{gathered}
$$

Proof. Representation (2.5) and the Taylor expansion give

$$
\left|\xi_{j}^{\varepsilon, 0}\right| \leq \frac{\tau^{2}}{6}\left\|\partial_{t}^{3} u\right\|_{L^{\infty}\left(0, T ; L^{\infty}(\Omega)\right)}+\frac{\tau h}{6}\left\|\partial_{x}^{3} \phi\right\|_{L^{\infty}(\Omega)} \lesssim h^{2}+\tau^{2}
$$

Similarly, we have

$$
\begin{aligned}
\left|\delta_{x}^{+} \xi_{j}^{\varepsilon, 0}\right| & \leq \frac{\tau^{2}}{6}\left\|\partial_{t}^{3} u^{\varepsilon}\right\|_{L^{\infty}\left(0, T ; W^{1, \infty}\right)}+\frac{\tau h}{6}\left\|\partial_{x}^{4} \phi\right\|_{L^{\infty}(\Omega)} \\
& \leq \frac{\tau^{2}}{6}\left\|\partial_{t}^{3} u^{\varepsilon}\right\|_{L^{\infty}\left(0, T ; H^{2}\right)}+\frac{\tau h}{6}\left\|\partial_{x}^{4} \phi\right\|_{L^{\infty}(\Omega)} \\
& \lesssim h^{2}+\tau^{2}, \quad j \in \mathscr{T}_{N} .
\end{aligned}
$$

Therefore,

$$
\left\|\xi^{\varepsilon, 0}\right\|_{H^{1}} \lesssim h^{2}+\tau^{2}
$$


Noting that

$$
\begin{aligned}
\xi_{j}^{\varepsilon, n}:= & \delta_{t}^{2} u^{\varepsilon}\left(x_{j}, t_{n}\right)-\frac{1}{2} \delta_{x}^{2}\left(u^{\varepsilon}\left(x_{j}, t_{n+1}\right)+u^{\varepsilon}\left(x_{j}, t_{n-1}\right)\right) \\
& +\frac{1}{2}\left(u^{\varepsilon}\left(x_{j}, t_{n+1}\right)+u^{\varepsilon}\left(x_{j}, t_{n-1}\right)\right)+u^{\varepsilon}\left(x_{j}, t_{n}\right) f_{\varepsilon}\left(\left(u^{\varepsilon}\left(x_{j}, t_{n}\right)\right)^{2}\right) \\
& -\left[\partial_{t t} u^{\varepsilon}\left(x_{j}, t_{n}\right)-\partial_{x x} u^{\varepsilon}\left(x_{j}, t_{n}\right)+u^{\varepsilon}\left(x_{j}, t_{n}\right)+u^{\varepsilon}\left(x_{j}, t_{n}\right) f_{\varepsilon}\left(\left(u^{\varepsilon}\left(x_{j}, t_{n}\right)\right)^{2}\right)\right] \\
= & {\left[\delta_{t}^{2} u^{\varepsilon}\left(x_{j}, t_{n}\right)-\partial_{t t} u^{\varepsilon}\left(x_{j}, t_{n}\right)\right]-\left[\frac{1}{2} \delta_{x}^{2}\left(u^{\varepsilon}\left(x_{j}, t_{n+1}\right)+u^{\varepsilon}\left(x_{j}, t_{n-1}\right)\right)-\partial_{x x} u^{\varepsilon}\left(x_{j}, t_{n}\right)\right] } \\
& +\frac{1}{2}\left(u^{\varepsilon}\left(x_{j}, t_{n+1}\right)+u^{\varepsilon}\left(x_{j}, t_{n-1}\right)\right)-u^{\varepsilon}\left(x_{j}, t_{n}\right)
\end{aligned}
$$

and using the Taylor expansion yields

$$
\xi_{j}^{\varepsilon, n}=\frac{\tau^{2}}{12} \alpha_{j}^{\varepsilon, n}+\frac{\tau^{2}}{2} \beta_{j}^{\varepsilon, n}+\frac{h^{2}}{12} \eta_{j}^{\varepsilon, n}+\frac{\tau^{2}}{2} \phi_{j}^{\varepsilon, n},
$$

where

$$
\begin{aligned}
\alpha_{j}^{\varepsilon, n} & =\int_{-1}^{1}(1-|s|)^{3} \partial_{t}^{4} u^{\varepsilon}\left(x_{j}, t_{n}+s \tau\right) d s \\
\beta_{j}^{\varepsilon, n} & =\int_{-1}^{1}(1-|s|) \partial_{t}^{2} u_{x x}^{\varepsilon}\left(x_{j}, t_{n}+s \tau\right) d s \\
\eta_{j}^{\varepsilon, n} & =\int_{-1}^{1}(1-|s|)^{3}\left(\partial_{x}^{4} u^{\varepsilon}\left(x_{j}+s h, t_{n+1}\right)+\partial_{x}^{4} u^{\varepsilon}\left(x_{j}+s h, t_{n-1}\right)\right) d s \\
\phi_{j}^{\varepsilon, n} & =\int_{-1}^{1}(1-|s|) \partial_{t}^{2} u^{\varepsilon}\left(x_{j}, t_{n}+s \tau\right) d s
\end{aligned}
$$

Applying the Cauchy-Schwarz inequality, we obtain

$$
\begin{aligned}
& \left\|\alpha^{\varepsilon, n}\right\|_{l^{2}}^{2} \\
= & h \sum_{j=1}^{N-1}\left|\alpha_{j}^{\varepsilon, n}\right|^{2} \leq h \int_{-1}^{1}(1-|s|)^{6} d s \sum_{j=1}^{N-1} \int_{-1}^{1}\left|\partial_{t}^{4} u^{\varepsilon}\left(x_{j}, t_{n}+s \tau\right)\right|^{2} d s \\
= & \frac{2}{7}\left[\int_{-1}^{1}\left\|\partial_{t}^{4} u^{\varepsilon}\left(\cdot, t_{n}+s \tau\right)\right\|_{L^{2}(\Omega)}^{2} d s-\int_{-1}^{1} \sum_{j=0}^{N-1} \int_{x_{j}}^{x_{j+1}} \int_{x_{j}}^{\omega} \partial_{x}\left|\partial_{t}^{4} u^{\varepsilon}\left(\hat{x}, t_{n}+s \tau\right)\right|^{2} d \hat{x} d \omega d s\right] \\
\leq & \frac{2}{7} \int_{-1}^{1}\left[\left\|\partial_{t}^{4} u^{\varepsilon}\left(\cdot, t_{n}+s \tau\right)\right\|_{L^{2}(\Omega)}^{2}+2 h\left\|\partial_{t}^{4} u_{x}^{\varepsilon}\left(\cdot, t_{n}+s \tau\right)\right\|_{L^{2}(\Omega)}\left\|\partial_{t}^{4} u^{\varepsilon}\left(\cdot, t_{n}+s \tau\right)\right\|_{L^{2}(\Omega)}\right] d s \\
\leq & \max _{0 \leq t \leq T}\left(\left\|\partial_{t}^{4} u^{\varepsilon}\right\|_{L^{2}(\Omega)}+h\left\|\partial_{t}^{4} u_{x}^{\varepsilon}\right\|_{L^{2}(\Omega)}\right)^{2} .
\end{aligned}
$$

Consequently, if $h \leq 1$, then

$$
\left\|\alpha^{\varepsilon, n}\right\|_{l^{2}} \leq\left\|\partial_{t}^{4} u^{\varepsilon}\right\|_{L^{\infty}\left(0, T ; H^{1}(\Omega)\right)}
$$


Similarly, we obtain

$$
\begin{aligned}
& \left\|\beta^{\varepsilon, n}\right\|_{l^{2}} \leq 2\left\|\partial_{t}^{2} u^{\varepsilon}\right\|_{L^{\infty}\left(0, T ; H^{3}(\Omega)\right)}, \\
& \left\|\phi^{\varepsilon, n}\right\|_{l^{2}} \leq\left\|\partial_{t}^{2} u^{\varepsilon}\right\|_{L^{\infty}\left(0, T ; H^{1}(\Omega)\right)} .
\end{aligned}
$$

On the other hand,

$$
\begin{aligned}
\left\|\eta^{\varepsilon, n}\right\|_{l^{2}}^{2} & \leq h \int_{-1}^{1}(1-|s|)^{6} d s \sum_{j=1}^{N-1} \int_{-1}^{1}\left|\partial_{x}^{4} u^{\varepsilon}\left(x_{j}+s h, t_{n+1}\right)+\partial_{x}^{4} u^{\varepsilon}\left(x_{j}+s h, t_{n-1}\right)\right|^{2} d s \\
& \leq \frac{4 h}{7} \sum_{j=1}^{N-1} \int_{-1}^{1}\left(\left|\partial_{x}^{4} u^{\varepsilon}\left(x_{j}+s h, t_{n+1}\right)\right|^{2}+\left|\partial_{x}^{4} u^{\varepsilon}\left(x_{j}+s h, t_{n-1}\right)\right|^{2}\right) d s \\
& \leq \frac{8}{7}\left(\left\|\partial_{x}^{4} u^{\varepsilon}\left(\cdot, t_{n-1}\right)\right\|_{L^{2}(\Omega)}^{2}+\left\|\partial_{x}^{4} u^{\varepsilon}\left(\cdot, t_{n+1}\right)\right\|_{L^{2}(\Omega)}^{2}\right) \\
& \leq 4\left\|u^{\varepsilon}\right\|_{L^{\infty}\left(0, T ; H^{4}(\Omega)\right)}^{2},
\end{aligned}
$$

so that $\left\|\eta^{\varepsilon, n}\right\|_{L^{2}} \leq 2\left\|u^{\varepsilon}\right\|_{L^{\infty}\left(0, T ; H^{4}(\Omega)\right)}$. Therefore, taking into account Condition 3.1, we get

$$
\begin{aligned}
\left\|\xi^{\varepsilon, n}\right\|_{L^{2}} \leq & \frac{\tau^{2}}{12}\left\|\partial_{t}^{4} u^{\varepsilon}\right\|_{L^{\infty}\left(0, T ; H^{1}(\Omega)\right)}+\tau^{2}\left\|\partial_{t}^{2} u^{\varepsilon}\right\|_{L^{\infty}\left(0, T ; H^{3}(\Omega)\right)} \\
& +\frac{h^{2}}{6}\left\|u^{\varepsilon}\right\|_{L^{\infty}\left(0, T ; H^{4}(\Omega)\right)}+\frac{\tau^{2}}{2}\left\|\partial_{t}^{2} u^{\varepsilon}\right\|_{L^{\infty}\left(0, T ; H^{1}(\Omega)\right)} \\
\lesssim & \tau^{2}+h^{2} .
\end{aligned}
$$

The same approach leads to the estimate

$$
\begin{aligned}
\left\|\delta_{x}^{+} \xi^{\varepsilon, n}\right\|_{l^{2}} \leq & \frac{\tau^{2}}{12}\left\|\partial_{t}^{4} u^{\varepsilon}\right\|_{L^{\infty}\left(0, T ; H^{2}(\Omega)\right)}+\tau^{2}\left\|\partial_{t}^{2} u^{\varepsilon}\right\|_{L^{\infty}\left(0, T ; H^{4}(\Omega)\right)} \\
& +\frac{h^{2}}{6}\left\|u^{\varepsilon}\right\|_{L^{\infty}\left(0, T ; H^{5}(\Omega)\right)}+\frac{\tau^{2}}{2}\left\|\partial_{t}^{2} u^{\varepsilon}\right\|_{L^{\infty}\left(0, T ; H^{2}(\Omega)\right)} \\
\lesssim & \tau^{2}+h^{2}
\end{aligned}
$$

and the proof is complete.

Subtracting (2.2) from (3.3), we obtain the equations

$$
\begin{aligned}
& \delta_{t}^{2} e_{j}^{\varepsilon, n}-\frac{1}{2} \delta_{x}^{2}\left(e_{j}^{\varepsilon, n+1}+e_{j}^{\varepsilon, n-1}\right)+\frac{1}{2}\left(e_{j}^{\varepsilon, n+1}+e_{j}^{\varepsilon, n-1}\right)=\xi_{j}^{\varepsilon, n}-\zeta_{j}^{\varepsilon, n}, \\
& e_{0}^{\varepsilon, n}=e_{N}^{\varepsilon, n}, \quad e_{-1}^{\varepsilon, n}=e_{N-1}^{\varepsilon, n}, \quad n=0,1, \ldots, \\
& e_{j}^{\varepsilon, 0}=0, \quad e_{j}^{\varepsilon, 1}=\tau \xi_{j}^{\varepsilon, 0}, \quad j \in \mathscr{T}_{N},
\end{aligned}
$$

where

$$
\zeta_{j}^{\varepsilon, n}=u^{\varepsilon}\left(x_{j}, t_{n}\right) f_{\varepsilon}\left(\left(u^{\varepsilon}\left(x_{j}, t_{n}\right)\right)^{2}\right)-u_{j}^{\varepsilon, n} f_{\varepsilon}\left(\left(u_{j}^{\varepsilon, n}\right)^{2}\right)
$$


We define the energy for the error vector $e^{\varepsilon, n}, n=0,1, \ldots$ as

$$
E_{e}^{n}:=\left\|\delta_{t}^{+} e^{\varepsilon, n}\right\|_{l^{2}}^{2}+\frac{1}{2}\left(\left\|\delta_{x}^{+} e^{\varepsilon, n+1}\right\|_{l^{2}}^{2}+\left\|\delta_{x}^{+} e^{\varepsilon, n}\right\|_{l^{2}}^{2}\right)+\frac{1}{2}\left(\left\|e^{\varepsilon, n+1}\right\|_{l^{2}}^{2}+\left\|e^{\varepsilon, n}\right\|_{l^{2}}^{2}\right) .
$$

Note that

$$
E_{e}^{0}:=\left\|\xi^{\varepsilon, 0}\right\|_{l^{2}}^{2}+\frac{\tau^{2}}{2}\left\|\delta_{x}^{+} \xi^{\varepsilon, 0}\right\|_{l^{2}}^{2}+\frac{\tau^{2}}{2}\left\|\xi^{\varepsilon, 0}\right\|_{l^{2}}^{2} \lesssim\left(\tau^{2}+h^{2}\right)^{2} .
$$

Proof of Theorem 3.1. It follows from Condition 3.1 and Lemma 3.1 that for sufficiently small $0<\tau<\tau_{1}$ and $0<h<h_{1}$, the first step discretisation error (2.5) can be estimated as

$$
e^{\varepsilon, 0}=0, \quad\left\|e^{\varepsilon, 1}\right\|_{H^{1}} \lesssim \tau^{2}+h^{2} .
$$

Thus for $n=0,1$ our claim is true.

Assume (3.1) is valid for all $n \leq m \leq(T / \tau)-1$ and show that it is also true for $n=m+1$. Denote

$$
\zeta_{j}^{\varepsilon, n}=u^{\varepsilon}\left(x_{j}, t_{n}\right) f_{\varepsilon}\left(\left(u^{\varepsilon}\left(x_{j}, t_{n}\right)\right)^{2}\right)-u_{j}^{\varepsilon, n} f_{\varepsilon}\left(\left(u_{j}^{\varepsilon, n}\right)^{2}\right) .
$$

If $\left|u_{j}^{\varepsilon, n}\right| \leq\left|u^{\varepsilon}\left(x_{j}, t_{n}\right)\right|$, then

$$
\begin{aligned}
\left|\zeta_{j}^{\varepsilon, n}\right|= & \mid u_{j}^{\varepsilon, n} \ln \left(\varepsilon^{2}+\left(u_{j}^{\varepsilon, n}\right)^{2}\right)-u_{j}^{\varepsilon, n} \ln \left(\varepsilon^{2}+\left(u^{\varepsilon}\left(x_{j}, t_{n}\right)\right)^{2}\right) \\
& \quad+u_{j}^{\varepsilon, n} \ln \left(\varepsilon^{2}+\left(u^{\varepsilon}\left(x_{j}, t_{n}\right)\right)^{2}\right)-u^{\varepsilon}\left(x_{j}, t_{n}\right) \ln \left(\varepsilon^{2}+\left(u^{\varepsilon}\left(x_{j}, t_{n}\right)\right)^{2}\right) \mid \\
= & \left|e_{j}^{\varepsilon, n} \ln \left(\varepsilon^{2}+\left(u^{\varepsilon}\left(x_{j}, t_{n}\right)\right)^{2}\right)+u_{j}^{\varepsilon, n} \ln \left(1+\frac{\left(u_{j}^{\varepsilon, n}\right)^{2}-\left(u^{\varepsilon}\left(x_{j}, t_{n}\right)\right)^{2}}{\varepsilon^{2}+\left(u^{\varepsilon}\left(x_{j}, t_{n}\right)\right)^{2}}\right)\right| \\
\leq & \left|e_{j}^{\varepsilon, n} \ln \left(\varepsilon^{2}+\left(u^{\varepsilon}\left(x_{j}, t_{n}\right)\right)^{2}\right)\right|+\left|u^{\varepsilon}\left(x_{j}, t_{n}\right) \frac{\left(u_{j}^{\varepsilon, n}\right)^{2}-\left(u^{\varepsilon}\left(x_{j}, t_{n}\right)\right)^{2}}{\left(u^{\varepsilon}\left(x_{j}, t_{n}\right)\right)^{2}}\right| \\
\leq & \left|e_{j}^{\varepsilon, n}\right| \max \left\{\ln \left(\frac{1}{\varepsilon^{2}}\right),\left|\ln \left(\Lambda^{2}+\varepsilon^{2}\right)\right|\right\}+2\left|e_{j}^{\varepsilon, n}\right| \\
= & \left|e_{j}^{\varepsilon, n}\right|\left(\max \left\{\ln \left(\frac{1}{\varepsilon^{2}}\right),\left|\ln \left(\Lambda^{2}+\varepsilon^{2}\right)\right|\right\}+2\right) .
\end{aligned}
$$

On the other hand, if $\left|u_{j}^{\varepsilon, n}\right| \geq\left|u^{\varepsilon}\left(x_{j}, t_{n}\right)\right|$, then

$$
\begin{aligned}
\left|\zeta_{j}^{\varepsilon, n}\right|= & \mid u^{\varepsilon}\left(x_{j}, t_{n}\right) \ln \left(\varepsilon^{2}+\left(u^{\varepsilon}\left(x_{j}, t_{n}\right)\right)^{2}\right)-u^{\varepsilon}\left(x_{j}, t_{n}\right) \ln \left(\varepsilon^{2}+\left(u_{j}^{\varepsilon, n}\right)^{2}\right) \\
& +u^{\varepsilon}\left(x_{j}, t_{n}\right) \ln \left(\varepsilon^{2}+\left(u_{j}^{\varepsilon, n}\right)^{2}\right)-u_{j}^{\varepsilon, n} \ln \left(\varepsilon^{2}+\left(u_{j}^{\varepsilon, n}\right)^{2}\right) \mid \\
= & \left|e_{j}^{\varepsilon, n} \ln \left(\varepsilon^{2}+\left(u_{j}^{\varepsilon, n}\right)^{2}\right)+u^{\varepsilon}\left(x_{j}, t_{n}\right) \ln \left(\frac{\varepsilon^{2}+\left(u^{\varepsilon}\left(x_{j}, t_{n}\right)\right)^{2}}{\varepsilon^{2}+\left(u_{j}^{\varepsilon, n}\right)^{2}}\right)\right|
\end{aligned}
$$




$$
\begin{aligned}
& \leq\left|e_{j}^{\varepsilon, n}\right| \max \left\{\ln \left(\frac{1}{\varepsilon^{2}}\right),\left|\ln \left((1+\Lambda)^{2}+\varepsilon^{2}\right)\right|\right\}+2\left|e_{j}^{\varepsilon, n}\right| \\
& =\left|e_{j}^{\varepsilon, n}\right|\left(\max \left\{\ln \left(\frac{1}{\varepsilon^{2}}\right),\left|\ln \left((1+\Lambda)^{2}+\varepsilon^{2}\right)\right|\right\}+2\right),
\end{aligned}
$$

where the assumption $\left\|u^{\varepsilon, n}\right\|_{l^{\infty}} \leq 1+\Lambda, n \leq m$ is used. Since $\varepsilon$ is sufficiently small, we have

$$
\left\|\zeta^{\varepsilon, n}\right\|_{l^{2}}^{2} \lesssim\left\|e^{\varepsilon, n}\right\|_{l^{2}}^{2}\left(\ln \varepsilon^{2}\right)^{2} .
$$

Multiplying (3.4a) by $h\left(e^{\varepsilon, n+1}-e^{\varepsilon, n-1}\right)$, then summing in $j \in \mathscr{T}_{N}$, and using the Young's inequality, Lemma 3.1, and the estimate (3.6), we obtain

$$
\begin{aligned}
E_{e}^{n}-E_{e}^{n-1} & =h \sum_{j=0}^{N-1}\left(\xi_{j}^{\varepsilon, n}-\zeta_{j}^{\varepsilon, n}\right)\left(e_{j}^{\varepsilon, n+1}-e_{j}^{\varepsilon, n-1}\right) \\
& \leq h \sum_{j=0}^{N-1}\left(\left|\xi_{j}^{\varepsilon, n}\right|+\left|\zeta_{j}^{\varepsilon, n}\right|\right)\left|e_{j}^{\varepsilon, n+1}-e_{j}^{\varepsilon, n-1}\right| \\
& \leq \tau\left(\left\|\xi^{\varepsilon, n}\right\|_{l^{2}}^{2}+\left\|\zeta^{\varepsilon, n}\right\|_{l^{2}}^{2}+\left\|\delta_{t}^{+} e^{\varepsilon, n}\right\|_{l^{2}}^{2}+\left\|\delta_{t}^{+} e^{\varepsilon, n-1}\right\|_{l^{2}}^{2}\right) \\
& \lesssim \tau\left(\left(\tau^{2}+h^{2}\right)^{2}+\left(\ln \varepsilon^{2}\right)^{2}\left\|e^{\varepsilon, n}\right\|_{l^{2}}^{2}+E_{e}^{n}+E_{e}^{n-1}\right), \quad 1 \leq n \leq \frac{T}{\tau}-1 .
\end{aligned}
$$

Therefore, there exists a sufficiently small constant $\tau_{2}>0$, such that if $0<\tau<\tau_{2}$, then

$$
E_{e}^{n}-E_{e}^{n-1} \lesssim \tau\left(\left(\tau^{2}+h^{2}\right)^{2}+\left(\ln \varepsilon^{2}\right)^{2}\left\|e^{\varepsilon, n}\right\|_{l^{2}}^{2}+E_{e}^{n-1}\right), \quad 1 \leq n \leq \frac{T}{\tau}-1 .
$$

Summing these inequalities in $n$ up to $m$ and recalling the estimate (3.5) gives

$$
E_{e}^{m} \lesssim\left(\tau^{2}+h^{2}\right)^{2}+\tau\left(\ln \varepsilon^{2}\right)^{2} \sum_{n=0}^{m-1} E_{e}^{n}, \quad 1 \leq m \leq \frac{T}{\tau}-1 .
$$

Applying the discrete Gronwall's inequality [28], we have

$$
E_{e}^{m} \lesssim e^{T\left(\ln \left(\varepsilon^{2}\right)\right)^{2}}\left(\tau^{2}+h^{2}\right)^{2}, \quad 1 \leq m \leq \frac{T}{\tau}-1 .
$$

Since for $0<\varepsilon \ll 1$, the estimate

$$
\left\|\delta_{x}^{+} e^{\varepsilon, m+1}\right\|_{l^{2}}^{2}+\left\|e^{\varepsilon, m+1}\right\|_{l^{2}}^{2} \leq 2 E_{e}^{m}
$$

holds and we obtain

$$
\left\|\delta_{x}^{+} e^{\varepsilon, m+1}\right\|_{l^{2}}^{2}+\left\|e^{\varepsilon, m+1}\right\|_{l^{2}}^{2} \lesssim e^{T\left(\ln \left(\varepsilon^{2}\right)\right)^{2}}\left(\tau^{2}+h^{2}\right)^{2} .
$$

Moreover, the Sobolev inequality implies

$$
\left\|e^{\varepsilon, m+1}\right\|_{l^{\infty}}^{2} \leq\left\|\delta_{x}^{+} e^{\varepsilon, m+1}\right\|_{l^{2}}^{2}+\left\|e^{\varepsilon, m+1}\right\|_{l^{2}}^{2} \lesssim e^{T\left(\ln \left(\varepsilon^{2}\right)\right)^{2}}\left(\tau^{2}+h^{2}\right)^{2} .
$$


Therefore, there are $\tau_{3}>0, h_{2}>0$ such that for $0<h<h_{2}, 0<\tau<\tau_{3}$, the triangle inequality gives

$$
\left\|u^{\varepsilon, m+1}\right\|_{l^{\infty}} \leq\left\|u^{\varepsilon}\left(\cdot, t_{m+1}\right)\right\|_{L^{\infty}(\Omega)}+\left\|e^{\varepsilon, m+1}\right\|_{l^{\infty}} \leq \Lambda+1 .
$$

Now we can set

$$
h_{0}:=\min \left\{h_{1}, h_{2}\right\}, \quad \tau_{0}=\min \left\{\tau_{1}, \tau_{2}, \tau_{3}\right\}
$$

\subsection{The proof of Theorem 3.2 for EXFD}

Define the local truncation error for the EXFD (2.3) by

$$
\begin{array}{r}
\xi_{j}^{\varepsilon, 0}:=\delta_{t}^{+} u^{\varepsilon}\left(x_{j}, 0\right)-\gamma\left(x_{j}\right)-\frac{\tau}{2}\left[\delta_{x}^{2} \phi\left(x_{j}\right)-\phi\left(x_{j}\right)-\phi\left(x_{j}\right) \ln \left(\varepsilon^{2}+\left(\phi\left(x_{j}\right)\right)^{2}\right)\right] \\
j \in \mathscr{T}_{N}, \\
\xi_{j}^{\varepsilon, n}:=\delta_{t}^{2} u^{\varepsilon}\left(x_{j}, t_{n}\right)-\delta_{x}^{2} u^{\varepsilon}\left(x_{j}, t_{n}\right)+u^{\varepsilon}\left(x_{j}, t_{n}\right)+u^{\varepsilon}\left(x_{j}, t_{n}\right) f_{\varepsilon}\left(\left(u^{\varepsilon}\left(x_{j}, t_{n}\right)\right)^{2}\right) \\
1 \leq n \leq \frac{T}{\tau}-1 .
\end{array}
$$

Lemma 3.2. Under Condition 3.1 the estimates

$$
\begin{gathered}
\left\|\xi^{\varepsilon, 0}\right\|_{H^{1}} \lesssim h^{2}+\tau^{2}, \quad\left\|\xi^{\varepsilon, n}\right\|_{l^{2}} \lesssim h^{2}+\tau^{2} \\
\left\|\delta_{x}^{+} \xi^{\varepsilon, n}\right\|_{l^{2}} \lesssim h^{2}+\tau^{2}, \quad 1 \leq n \leq \frac{T}{\tau}-1
\end{gathered}
$$

hold.

Proof. Lemma 3.1 shows that

$$
\left\|\xi^{\varepsilon, 0}\right\|_{H^{1}} \lesssim h^{2}+\tau^{2}
$$

Since

$$
\begin{aligned}
\xi_{j}^{\varepsilon, n}= & \delta_{t}^{2} u^{\varepsilon}\left(x_{j}, t_{n}\right)-\delta_{x}^{2} u^{\varepsilon}\left(x_{j}, t_{n}\right)+u^{\varepsilon}\left(x_{j}, t_{n}\right)+u^{\varepsilon}\left(x_{j}, t_{n}\right) f_{\varepsilon}\left(\left(u^{\varepsilon}\left(x_{j}, t_{n}\right)\right)^{2}\right) \\
& -\left[\partial_{t} u^{\varepsilon}\left(x_{j}, t_{n}\right)-\partial_{x x} u^{\varepsilon}\left(x_{j}, t_{n}\right)+u^{\varepsilon}\left(x_{j}, t_{n}\right)+u^{\varepsilon}\left(x_{j}, t_{n}\right) f_{\varepsilon}\left(\left(u^{\varepsilon}\left(x_{j}, t_{n}\right)\right)^{2}\right)\right] \\
= & {\left[\delta_{t}^{2} u^{\varepsilon}\left(x_{j}, t_{n}\right)-\partial_{t t} u^{\varepsilon}\left(x_{j}, t_{n}\right)\right]-\left[\delta_{x}^{2} u^{\varepsilon}\left(x_{j}, t_{n}\right)-\partial_{x x} u^{\varepsilon}\left(x_{j}, t_{n}\right)\right], }
\end{aligned}
$$

we can use the Taylor expansion to obtain

$$
\xi_{j}^{\varepsilon, n}=\frac{\tau^{2}}{12} \alpha_{j}^{\varepsilon, n}+\frac{h^{2}}{6} \beta_{j}^{\varepsilon, n},
$$

where

$$
\alpha_{j}^{\varepsilon, n}=\int_{-1}^{1}(1-|s|)^{3} \partial_{t}^{4} u^{\varepsilon}\left(x_{j}, t_{n}+s \tau\right) d s, \quad \beta_{j}^{\varepsilon, n}=\int_{-1}^{1}(1-|s|)^{3} \partial_{x}^{3} u^{\varepsilon}\left(x_{j}+s h, t_{n}\right) d s .
$$


The Cauchy-Schwarz inequality implies

$$
\begin{aligned}
& \left\|\alpha^{\varepsilon, n}\right\|_{l^{2}}^{2} \\
= & h \sum_{j=1}^{N-1}\left|\alpha_{j}^{\varepsilon, n}\right|^{2} \leq h \int_{-1}^{1}(1-|s|)^{6} d s \sum_{j=1}^{N-1} \int_{-1}^{1}\left|\partial_{t}^{4} u^{\varepsilon}\left(x_{j}, t_{n}+s \tau\right)\right|^{2} d s \\
= & \frac{2}{7}\left[\int_{-1}^{1}\left\|\partial_{t}^{4} u^{\varepsilon}\left(\cdot, t_{n}+s \tau\right)\right\|_{L^{2}(\Omega)}^{2} d s-\int_{-1}^{1} \sum_{j=0}^{N-1} \int_{x_{j}}^{x_{j+1}} \int_{x_{j}}^{\omega} \partial_{x}\left|\partial_{t}^{4} u^{\varepsilon}\left(\hat{x}, t_{n}+s \tau\right)\right|^{2} d \hat{x} d \omega d s\right] \\
\leq & \frac{2}{7} \int_{-1}^{1}\left[\left\|\partial_{t}^{4} u^{\varepsilon}\left(\cdot, t_{n}+s \tau\right)\right\|_{L^{2}(\Omega)}^{2}+2 h\left\|\partial_{t}^{4} \partial_{x} u^{\varepsilon}\left(\cdot, t_{n}+s \tau\right)\right\|_{L^{2}(\Omega)}\left\|\partial_{t}^{4} u^{\varepsilon}\left(\cdot, t_{n}+s \tau\right)\right\|_{L^{2}(\Omega)}\right] d s \\
\leq & \max _{0 \leq t \leq T}\left(\left\|\partial_{t}^{4} u^{\varepsilon}\right\|_{L^{2}(\Omega)}+h\left\|\partial_{t}^{4} \partial_{x} u^{\varepsilon}\right\|_{L^{2}(\Omega)}\right)^{2},
\end{aligned}
$$

so that if $h \leq 1$, then

$$
\left\|\alpha^{\varepsilon, n}\right\|_{l^{2}} \leq\left\|\partial_{t}^{4} u^{\varepsilon}\right\|_{L^{\infty}\left(0, T ; H^{1}(\Omega)\right)}
$$

On the other hand, we can write

$$
\begin{aligned}
\left\|\beta^{\varepsilon, n}\right\|_{l^{2}}^{2} & \leq h \int_{-1}^{1}(1-|s|)^{6} d s \sum_{j=1}^{N-1} \int_{-1}^{1}\left|\partial_{x}^{3} u^{\varepsilon}\left(x_{j}+s h, t_{n}\right)\right|^{2} d s \\
& \leq \frac{2 h}{7} \sum_{j=1}^{N-1} \int_{-1}^{1}\left|\partial_{x}^{3} u^{\varepsilon}\left(x_{j}+s h, t_{n}\right)\right|^{2} d s \\
& \leq \frac{4}{7}\left(\left\|\partial_{x}^{3} u^{\varepsilon}\left(\cdot, t_{n}\right)\right\|_{L^{2}(\Omega)}^{2}\right) \\
& \leq\left\|u^{\varepsilon}\right\|_{L^{\infty}\left(0, T ; H^{3}(\Omega)\right)}^{2} .
\end{aligned}
$$

Therefore, Condition 3.1 yields

$$
\begin{aligned}
\left\|\xi^{\varepsilon, n}\right\|_{l^{2}} & \leq \frac{\tau^{2}}{12}\left\|\partial_{t}^{4} u^{\varepsilon}\right\|_{L^{\infty}\left(0, T ; H^{1}(\Omega)\right)}+\frac{h^{2}}{6}\left\|u^{\varepsilon}\right\|_{L^{\infty}\left(0, T ; H^{3}(\Omega)\right)} \\
& \lesssim \tau^{2}+h^{2} .
\end{aligned}
$$

Similar approach shows that

$$
\begin{aligned}
\left\|\delta_{x}^{+} \xi^{\varepsilon, n}\right\|_{l^{2}} & \leq \frac{\tau^{2}}{12}\left\|\partial_{t}^{4} u^{\varepsilon}\right\|_{L^{\infty}\left(0, T ; H^{2}(\Omega)\right)}+\frac{h^{2}}{6}\left\|u^{\varepsilon}\right\|_{L^{\infty}\left(0, T ; H^{4}(\Omega)\right)} \\
& \lesssim \tau^{2}+h^{2}
\end{aligned}
$$

Subtracting (2.3) from (3.7) leads to the following relations for the error $e_{j}^{\varepsilon, n}$ :

$$
\begin{aligned}
& \delta_{t}^{2} e_{j}^{\varepsilon, n}-\delta_{x}^{2} e_{j}^{\varepsilon, n}+e_{j}^{\varepsilon, n}=\xi_{j}^{\varepsilon, n}-\zeta_{j}^{\varepsilon, n}, \\
& e_{0}^{\varepsilon, n}=e_{N}^{\varepsilon, n}, \quad e_{-1}^{\varepsilon, n}=e_{N-1}^{\varepsilon, n}, \quad n=0,1, \ldots, \\
& e_{j}^{\varepsilon, 0}=0, \quad e_{j}^{\varepsilon, 1}=\tau \xi_{j}^{\varepsilon, 0}, \quad j \in \mathscr{T}_{N},
\end{aligned}
$$


where

$$
\zeta_{j}^{\varepsilon, n}=u^{\varepsilon}\left(x_{j}, t_{n}\right) f_{\varepsilon}\left(\left(u^{\varepsilon}\left(x_{j}, t_{n}\right)\right)^{2}\right)-u_{j}^{\varepsilon, n} f_{\varepsilon}\left(\left(u_{j}^{\varepsilon, n}\right)^{2}\right) .
$$

Defining the energy of the error vector $e^{\varepsilon, n}, n=0,1, \ldots$ as

$$
\begin{aligned}
E_{e}^{n}:= & \left(1-\frac{\tau^{2}}{2}-\frac{\tau^{2}}{h^{2}}\right)\left\|\delta_{t}^{+} e^{\varepsilon, n}\right\|_{l^{2}}^{2}+\frac{1}{2}\left(\left\|e^{\varepsilon, n}\right\|_{l^{2}}^{2}+\left\|e^{\varepsilon, n+1}\right\|_{l^{2}}^{2}\right) \\
& +\frac{1}{2 h} \sum_{j=0}^{N-1}\left[\left(e_{j+1}^{\varepsilon, n+1}-e_{j}^{\varepsilon, n}\right)^{2}+\left(e_{j+1}^{\varepsilon, n}-e_{j}^{\varepsilon, n+1}\right)^{2}\right],
\end{aligned}
$$

we obtain

$$
\begin{aligned}
E_{e}^{0} & :=\left(1-\frac{\tau^{2}}{2}-\frac{\tau^{2}}{h^{2}}\right)\left\|\delta_{t}^{+} e^{\varepsilon, 0}\right\|_{l^{2}}^{2}+\left(\frac{1}{2}+\frac{1}{h^{2}}\right)\left\|e^{\varepsilon, 1}\right\|_{l^{2}}^{2} \\
& =\left\|\xi^{\varepsilon, 0}\right\|_{l^{2}}^{2} \lesssim\left(\tau^{2}+h^{2}\right)^{2} .
\end{aligned}
$$

Proof of Theorem 3.2. If $n=1$, Condition 3.1 and Lemma 3.1 show that for sufficiently small $0<\tau<\tau_{1}$ and $0<h<h_{1}$ the first step error of the discretisation (2.5) is

$$
e^{\varepsilon, 0}=0, \quad\left\|e^{\varepsilon, 1}\right\|_{H^{1}} \lesssim \tau^{2}+h^{2} .
$$

Thus for $n=0,1$ the estimate is true. Assume that (3.1) is valid for all $n \leq m \leq(T / \tau)-1$ and show it holds for $n=m+1$. Setting

$$
\zeta_{j}^{\varepsilon, n}:=u^{\varepsilon}\left(x_{j}, t_{n}\right) f_{\varepsilon}\left(\left(u^{\varepsilon}\left(x_{j}, t_{n}\right)\right)^{2}\right)-u_{j}^{\varepsilon, n} f_{\varepsilon}\left(\left(u_{j}^{\varepsilon, n}\right)^{2}\right)
$$

and using the same approach as in the proof of Theorem 3.1, we have

$$
\left\|\zeta^{\varepsilon, n}\right\|_{l^{2}}^{2} \lesssim\left\|e^{\varepsilon, n}\right\|_{l^{2}}^{2}\left(\ln \varepsilon^{2}\right)^{2} .
$$

Multiplying (3.8a) by $h\left(e^{\varepsilon, n+1}-e^{\varepsilon, n-1}\right)$, summing the results in $j \in \mathscr{T}_{N}$, and using the Young's inequality, Lemma 3.1, [8, Lemma 2] and (3.9), (3.11) gives

$$
\begin{aligned}
E_{e}^{n}-E_{e}^{n-1} & =h \sum_{j=0}^{N-1}\left(\xi_{j}^{\varepsilon, n}-\zeta_{j}^{\varepsilon, n}\right)\left(e_{j}^{\varepsilon, n+1}-e_{j}^{\varepsilon, n-1}\right) \\
& \leq h \sum_{j=0}^{N-1}\left(\left|\xi_{j}^{\varepsilon, n}\right|+\left|\zeta_{j}^{\varepsilon, n}\right|\right)\left|e_{j}^{\varepsilon, n+1}-e_{j}^{\varepsilon, n-1}\right| \\
& \leq \tau\left(\left\|\xi^{\varepsilon, n}\right\|_{l^{2}}^{2}+\left\|\zeta^{\varepsilon, n}\right\|_{l^{2}}^{2}+\left\|\delta_{t}^{+} e^{\varepsilon, n}\right\|_{l^{2}}^{2}+\left\|\delta_{t}^{+} e^{\varepsilon, n-1}\right\|_{l^{2}}^{2}\right) \\
& \lesssim \tau\left(\left(\tau^{2}+h^{2}\right)^{2}+\left(\ln \varepsilon^{2}\right)^{2}\left\|e^{\varepsilon, n}\right\|_{l^{2}}^{2}+E_{e}^{n}+E_{e}^{n-1}\right), \quad 1 \leq n \leq \frac{T}{\tau}-1 .
\end{aligned}
$$

Therefore, there is a constant $\tau_{2}>0$, such that for all $0<\tau<\tau_{2}$ the inequality

$$
E_{e}^{n}-E_{e}^{n-1} \lesssim \tau\left(\left(\tau^{2}+h^{2}\right)^{2}+\left(\ln \varepsilon^{2}\right)^{2}\left\|e^{\varepsilon, n}\right\|_{l^{2}}^{2}+E_{e}^{n-1}\right), \quad 1 \leq n \leq \frac{T}{\tau}-1
$$


holds. Summing these inequalities in $n$ and exploiting (3.10) yields

$$
E_{e}^{m} \lesssim\left(\tau^{2}+h^{2}\right)^{2}+\tau\left(\ln \varepsilon^{2}\right)^{2} \sum_{n=0}^{m-1} E_{e}^{n}, \quad 1 \leq m \leq \frac{T}{\tau}-1 .
$$

By the discrete Gronwall's inequality [28], we write

$$
E_{e}^{m} \lesssim e^{T\left(\ln \left(\varepsilon^{2}\right)\right)^{2}}\left(\tau^{2}+h^{2}\right)^{2}, \quad 1 \leq m \leq \frac{T}{\tau}-1 .
$$

In addition, if $\tau \leq(1 / 2) \min \{1, h\}$, then $1-\tau^{2} / 2-\tau^{2} / h^{2} \geq 1 / 4>0$, and it follows from

$$
\begin{aligned}
\left\|\delta_{x}^{+} e^{m+1}\right\|_{l^{2}}^{2} & =\frac{1}{h} \sum_{j=0}^{N-1}\left(e_{j+1}^{m+1}-e_{j}^{m}-\tau \delta_{t}^{+} e_{j}^{m}\right)^{2} \\
& \leq \frac{2}{h} \sum_{j=0}^{N-1}\left(e_{j+1}^{m+1}-e_{j}^{m}\right)^{2}+\frac{2 \tau^{2}}{h^{2}}\left\|\delta_{t}^{+} e^{m}\right\|_{l^{2}}^{2}
\end{aligned}
$$

that

and, consequently,

$$
E_{e}^{m} \geq \frac{1}{4}\left\|\delta_{x}^{+} e^{m+1}\right\|_{l^{2}}^{2}+\frac{1}{4}\left\|e^{m+1}\right\|_{l^{2}}^{2}
$$

$$
\left\|\delta_{x}^{+} e^{\varepsilon, m+1}\right\|_{l^{2}}^{2}+\left\|e^{\varepsilon, m+1}\right\|_{l^{2}}^{2} \lesssim e^{T\left(\ln \left(\varepsilon^{2}\right)\right)^{2}}\left(\tau^{2}+h^{2}\right)^{2} .
$$

The Sobolev inequality implies

$$
\left\|e^{\varepsilon, m+1}\right\|_{l^{\infty}}^{2} \leq\left\|\delta_{x}^{+} e^{\varepsilon, m+1}\right\|_{l^{2}}^{2}+\left\|e^{\varepsilon, m+1}\right\|_{l^{2}}^{2} \lesssim e^{T\left(\ln \left(\varepsilon^{2}\right)\right)^{2}}\left(\tau^{2}+h^{2}\right)^{2} .
$$

Therefore, there exist $\tau_{3}>0, h_{2}>0$ such that for all $0<h<h_{2}, 0<\tau<\tau_{3}$, we have

$$
\left\|u^{\varepsilon, m+1}\right\|_{l^{\infty}} \leq\left\|u^{\varepsilon}\left(\cdot, t_{m+1}\right)\right\|_{L^{\infty}(\Omega)}+\left\|e^{\varepsilon, m+1}\right\|_{l^{\infty}} \leq \Lambda+1 .
$$

We complete the proof by choosing $h_{0}:=\min \left\{h_{1}, h_{2}\right\}, \tau_{0}:=\min \left\{\tau_{1}, \tau_{2}, \tau_{3}\right\}$.

\section{Numerical Results}

In this section, we present the results of numerical experiments related to the EXFD (2.3) scheme. Since SIFD scheme (2.2) behaves similar to EXFD scheme (2.3), we omit the details here. Let $d=1, \lambda=1$ and the errors are defined as

$$
\hat{e}^{\varepsilon}\left(t_{n}\right):=u\left(\cdot, t_{n}\right)-u^{\varepsilon}\left(\cdot, t_{n}\right), \quad e^{\varepsilon}\left(t_{n}\right):=u^{\varepsilon}\left(\cdot, t_{n}\right)-u^{\varepsilon, n}, \quad \tilde{e}^{\varepsilon}\left(t_{n}\right):=u\left(\cdot, t_{n}\right)-u^{\varepsilon, n},
$$

where $u, u^{\varepsilon}$ are the exact solutions of the LogKGE (1.1) and the RLogKGE (1.2), and $u^{n}$ and $u^{\varepsilon, n}$ are, respectively, the numerical solutions of the Eqs. (1.1) and (1.2).

The above residues are estimated in various norms, and we use the following notations:

$$
\begin{aligned}
e_{\infty}^{\varepsilon}\left(t_{n}\right) & :=\left\|u^{\varepsilon}\left(\cdot, t_{n}\right)-u^{\varepsilon, n}\right\|_{l^{\infty}}, \quad e_{2}^{\varepsilon}\left(t_{n}\right):=\left\|u^{\varepsilon}\left(\cdot, t_{n}\right)-u^{\varepsilon, n}\right\|_{l^{2}}, \\
e_{H^{1}}^{\varepsilon}\left(t_{n}\right) & :=\sqrt{\left(e_{2}^{\varepsilon}\left(t_{n}\right)\right)^{2}+\left\|\delta_{x}^{+}\left(u^{\varepsilon}\left(\cdot, t_{n}\right)-u^{\varepsilon, n}\right)\right\|_{l^{2}}^{2}} .
\end{aligned}
$$


Example 4.1. Let

$$
\phi(x)=e^{-x^{2} / 6}, \quad \gamma(x)=\frac{2 x}{3} e^{-x^{2} / 6}
$$

and

$$
u(x, t)=e^{-(x-2 t)^{2} / 6}
$$

is the Gaussian solitary wave solution. The RLogKGE (1.2) is considered on the domain $\Omega=[-16,16]$. The exact solution $u^{\varepsilon}$ is determined by the EXFD scheme (2.3) with $\varepsilon=$ $10^{-7}$.

Example 4.2. Let

$$
\phi(x)=\frac{2}{e^{-x^{2}}+e^{x^{2}}}, \quad \gamma(x)=0 .
$$

The computation domain is $\Omega=[-16,16]$ with periodic boundary conditions. Since an analytical solution is not known, we use the reference solution $u^{\varepsilon}$ obtained by EXFD (2.3) with mesh size $h=2^{-10}$ and time step $\tau=0.01 \times 2^{-9}$. The reference solution $u$ is approximated by $u^{\varepsilon}$ with $\varepsilon=10^{-7}$.

\subsection{Convergence of the regularised model}

Here we test the accuracy of the regularised model. Fig. 1 represents $\left\|\hat{e}^{\varepsilon}\right\|_{l^{2}},\left\|\hat{e}^{\varepsilon}\right\|_{l^{\infty}}$, $\left\|\hat{e}^{\varepsilon}\right\|_{H^{1}}$ with the scheme EXFD (2.3) for Examples 4.1 and 4.2. The exact solution $u$ is computed by employing refined time step $\tau_{e}=0.01 \times 2^{-9}$ and mesh size $h_{e}=2^{-10}$. The errors are displayed at $T=0.5$.

Fig. 1 shows that the solutions of the RLogKGE (1.2) converge linearly to the LogKGE (1.1) with respect to $\varepsilon$. The convergence is $\mathscr{O}(\varepsilon)$ in each of $l^{2}$-, $l^{\infty}$-, and $H^{1}$-norms.
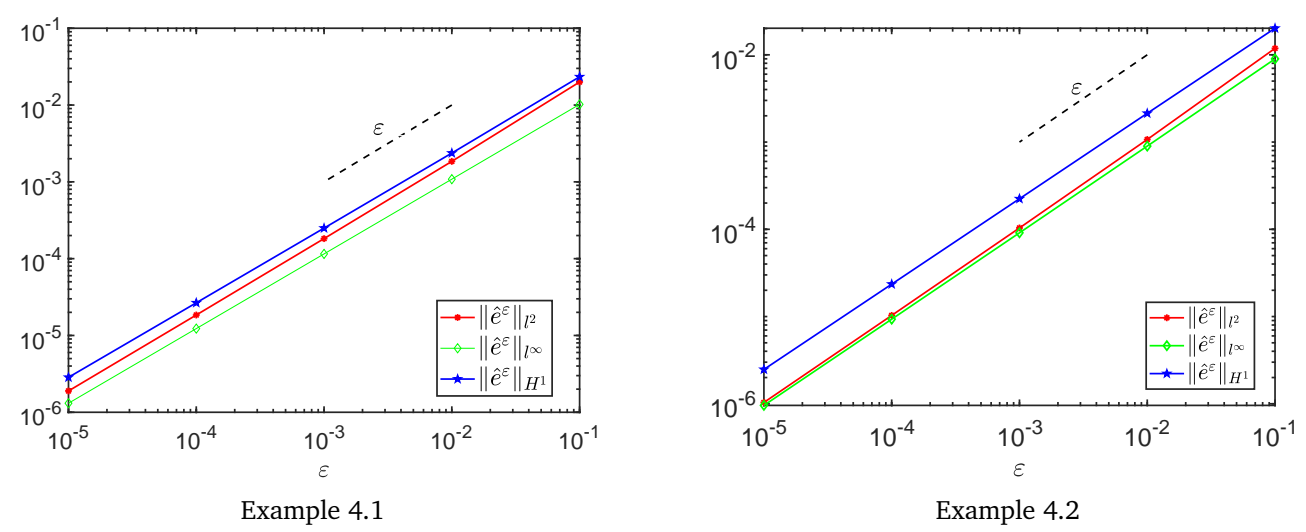

Figure 1: Errors $\hat{e}^{\varepsilon}(0.5)$ in different norms for scheme (2.3). 


\subsection{Convergence of FDTD for RLogKGE}

Consider the convergence of the scheme (2.3) for the Eq. (1.2) for various meshes and time steps $\tau$ for a fixed parameter $0<\varepsilon \ll 1$.

Firstly, we deal with temporal errors in $l^{2}$-, $l^{\infty}$-, and $H^{1}$-norms at $T=1$, cf. Figs. 2 and 3. Following the stability condition for the scheme (2.3), we set $0<\tau<\min \{1 / 2, h / 2\}$, simultaneously varying the mesh size and time step as $\tau_{j}=0.01 \times 2^{-j}, h_{j}=2^{-j}$ for $j=$ $1, \ldots, 7$.

Secondly, to check the spatial accuracy of the EXFD (2.3) at $T=1$, we set time step $\tau=\tau_{e}=0.01 \times 2^{-9}$, so that the errors arising from time discretisation are ignored. Solve the RLogKGE (1.2) with the FDTD. The results are displayed in Figs. 4 and 5. Figs. 4 and 5 demonstrate $\left\|e^{\varepsilon}\right\|_{l^{2}},\left\|e^{\varepsilon}\right\|_{l^{\infty}},\left\|e^{\varepsilon}\right\|_{H^{1}}$ for different $h$ for the EXFD scheme (2.3).

Figs. 2-5 show that for the Eq. (1.2), method (2.3) converges quadratically - i.e. as $\mathscr{O}\left(\tau^{2}+h^{2}\right)$.

\subsection{Convergence of FDTD to the LogKGE}

We check the convergence rates of the finite difference schemes SIFD (2.2) and EXFD (2.3) for the LogKGE (1.1) in Example 4.1. Tables 1 and 2 display $l^{2}$-norm, $l^{\infty}$-norm, $H^{1}$-norm of $\tilde{e}^{\varepsilon}(1)$, respectively, for various mesh size $h$, time step $\tau$ and parameter $\varepsilon$.

Tables 1 and 2 show that the schemes SIFD (2.2), EXFD (2.3) are uniformly second order in space and time only if $\varepsilon \lesssim \tau^{2}, \varepsilon \lesssim h^{2}$, cf. lower triangles below the diagonals in bold letter. If $\tau^{2} \lesssim \varepsilon, h^{2} \lesssim \varepsilon$, the (1.2) converges to the Eq.(1.1) linearly as $\mathscr{O}(\varepsilon)$, cf. the far right column.

\subsection{The evolution of the solution}

Fig. 6 shows numerical solutions of (2.3) for Example 4.2 at time $T=1,5,9$. The step size is $\tau=0.01 \times 2^{-7}$ and the mesh size $h=2^{-7}$ for the larger domain [-20,20]. For different $\varepsilon$, numerical solutions for the two schemes are very close at fixed times. If time goes by, waves spread outward and new waves appear. We can conclude that the both discrete schemes are stable.

\section{Conclusions}

In order to deal with the origin singularity of the LogKGE (1.1), we use the Eq. (1.2) with a small regularisation parameter $0<\varepsilon \ll 1$. Two finite difference methods - viz. SIFD and EXFD for the Eq. (1.2), are studied. It is proven that they have the second order of accuracy both in space and time. Numerical experiments show that the solutions of the Eq. (1.2) converge to the solution of the Eq. (1.1) as $\mathscr{O}(\varepsilon)$ and that the errors of FDTD methods depend on $\tau, h, \varepsilon$. 

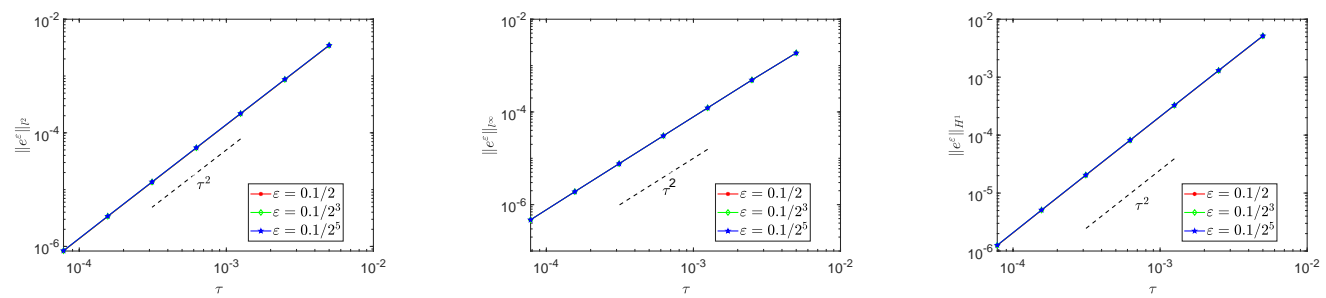

Figure 2: Example 4.1. Temporal errors $e^{\varepsilon}(1)$ in different norms.
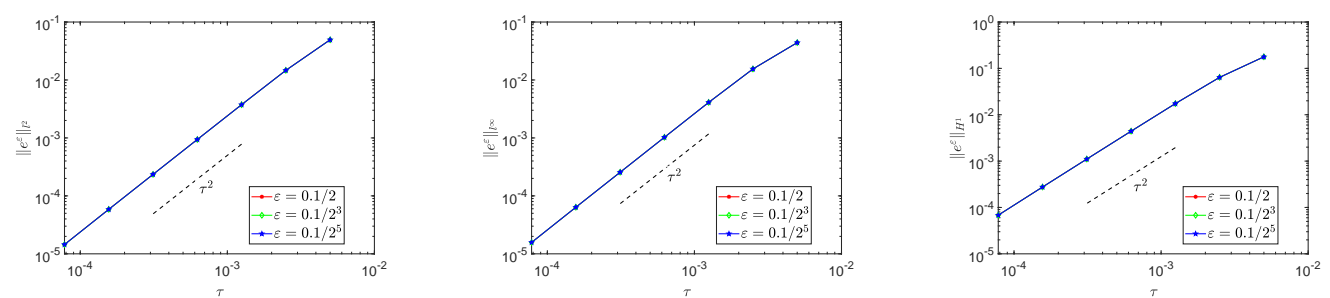

Figure 3: Example 4.2. Temporal errors $e^{\varepsilon}(1)$ in different norms.
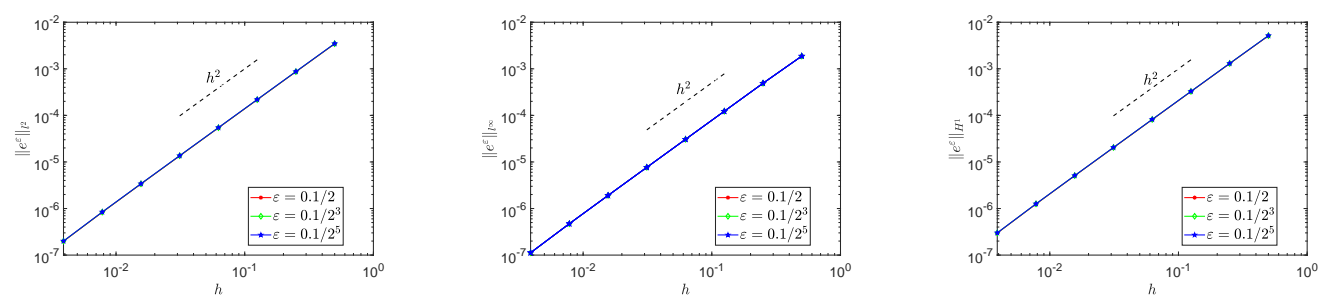

Figure 4: Example 4.1. Spatial errors $e^{\varepsilon}(1)$ in different norms.
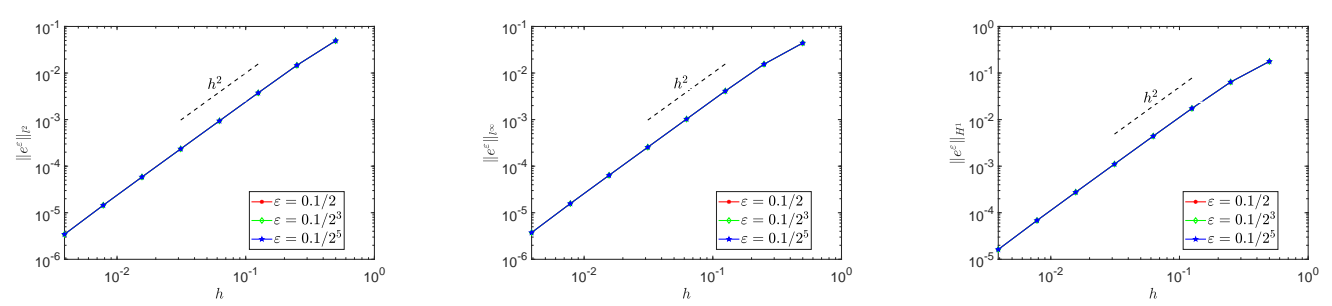

Figure 5: Example 4.2. Spatial errors $e^{\varepsilon}(1)$ in different norms.
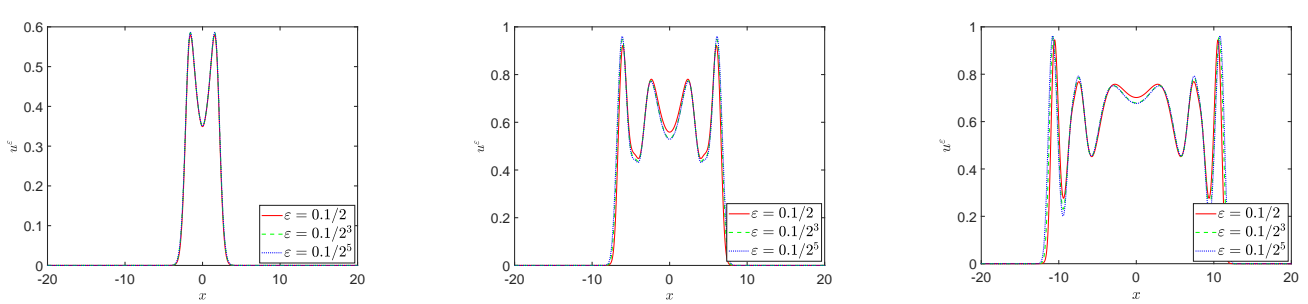

Figure 6: Numerical solution $u^{\varepsilon}$ obtained by the scheme (2.3). 
Table 1: Convergence of SIFD scheme (2.2) to LogKGE (1.1).

\begin{tabular}{|c|c|c|c|c|c|c|}
\hline$\left\|\tilde{e}^{\varepsilon}(1)\right\|_{l^{\infty}}$ & $\begin{aligned} h & =0.1 \\
\tau & =0.1\end{aligned}$ & $\begin{array}{l}h / 2 \\
\tau / 2\end{array}$ & $\begin{array}{l}h / 2^{2} \\
\tau / 2^{2}\end{array}$ & $\begin{array}{l}h / 2^{3} \\
\tau / 2^{3}\end{array}$ & $\begin{array}{l}h / 2^{4} \\
\tau / 2^{4}\end{array}$ & $\begin{array}{l}h / 2^{5} \\
\tau / 2^{5}\end{array}$ \\
\hline$\varepsilon=10^{-3}$ & 4.03E-03 & $1.03 \mathrm{E}-03$ & 7.34E-04 & 7.66E-04 & 7.74E-04 & 7.76E-04 \\
\hline rate & - & 1.97 & 0.48 & -0.06 & -0.02 & 0.00 \\
\hline$\varepsilon / 4$ & 4.03E-03 & $1.03 \mathrm{E}-03$ & 2.59E-04 & $2.24 \mathrm{E}-04$ & $2.28 \mathrm{E}-04$ & $2.29 \mathrm{E}-04$ \\
\hline rate & - & 1.97 & 1.99 & 0.21 & -0.03 & -0.01 \\
\hline$\varepsilon / 4^{2}$ & 4.03E-03 & $1.03 \mathrm{E}-03$ & $2.59 \mathrm{E}-04$ & 6.52E-05 & $6.70 \mathrm{E}-05$ & 6.75E-05 \\
\hline rate & - & 1.97 & 1.99 & 1.99 & -0.04 & -0.01 \\
\hline$\varepsilon / 4^{3}$ & 4.03E-03 & $1.03 \mathrm{E}-03$ & 2.59E-04 & $6.50 \mathrm{E}-05$ & $1.96 \mathrm{E}-05$ & $1.98 \mathrm{E}-05$ \\
\hline rate & - & 1.97 & 1.99 & 1.99 & 1.73 & -0.01 \\
\hline$\varepsilon / 4^{4}$ & 4.03E-03 & 1.03E-03 & $2.59 \mathrm{E}-04$ & $6.50 \mathrm{E}-05$ & $1.63 \mathrm{E}-05$ & $5.81 \mathrm{E}-06$ \\
\hline rate & - & 1.97 & 1.99 & 1.99 & 2.00 & 1.49 \\
\hline \multirow[t]{2}{*}{$\left\|\tilde{e}^{\varepsilon}(1)\right\|_{l^{2}}$} & $h=0.1$ & $h / 2$ & $h / 2^{2}$ & $h / 2^{3}$ & $h / 2^{4}$ & $h / 2^{5}$ \\
\hline & $\tau=0.1$ & $\tau / 2$ & $\tau / 2^{2}$ & $\tau / 2^{3}$ & $\tau / 2^{4}$ & $\tau / 2^{5}$ \\
\hline$\varepsilon=10^{-3}$ & 7.72E-03 & $2.23 \mathrm{E}-03$ & $1.30 \mathrm{E}-03$ & $1.24 \mathrm{E}-03$ & $1.25 \mathrm{E}-03$ & $1.25 \mathrm{E}-03$ \\
\hline rate & - & 1.80 & 0.78 & 0.06 & -0.01 & 0.00 \\
\hline$\varepsilon / 4$ & 7.73E-03 & $1.99 \mathrm{E}-03$ & 5.93E-04 & 3.69E-04 & $3.56 \mathrm{E}-04$ & $3.56 \mathrm{E}-04$ \\
\hline rate & - & 1.96 & 1.75 & 0.68 & 0.05 & 0.00 \\
\hline$\varepsilon / 4^{2}$ & 7.74E-03 & $1.98 \mathrm{E}-03$ & 5.07E-04 & 1.59E-04 & $1.05 \mathrm{E}-04$ & $1.02 \mathrm{E}-04$ \\
\hline rate & - & 1.97 & 1.97 & 1.68 & 0.59 & 0.05 \\
\hline$\varepsilon / 4^{3}$ & 7.74E-03 & $1.98 \mathrm{E}-03$ & $5.02 \mathrm{E}-04$ & $1.29 \mathrm{E}-04$ & $4.25 \mathrm{E}-05$ & 3.01E-05 \\
\hline rate & - & 1.96 & 1.98 & 1.96 & 1.60 & 0.50 \\
\hline$\varepsilon / 4^{4}$ & 7.74E-03 & $1.98 \mathrm{E}-03$ & 5.01E-04 & $1.26 \mathrm{E}-04$ & $3.26 \mathrm{E}-05$ & $1.14 \mathrm{E}-05$ \\
\hline rate & - & 1.96 & 1.98 & 1.99 & 1.95 & 1.51 \\
\hline \multirow[t]{2}{*}{$\left\|\tilde{e}^{\varepsilon}(1)\right\|_{H^{1}}$} & $h=0.1$ & $h / 2$ & $\overline{h / 2^{2}}$ & $h / 2^{3}$ & $h / 2^{4}$ & $h / 2^{5}$ \\
\hline & $\tau=0.1$ & $\tau / 2$ & $\tau / 2^{2}$ & $\tau / 2^{3}$ & $\tau / 2^{4}$ & $\tau / 2^{5}$ \\
\hline$\varepsilon=10^{-3}$ & $1.08 \mathrm{E}-02$ & 3.07E-03 & $1.68 \mathrm{E}-03$ & 1.59E-03 & $1.59 \mathrm{E}-03$ & $1.59 \mathrm{E}-03$ \\
\hline rate & - & 1.81 & 0.87 & 0.09 & 0.00 & 0.00 \\
\hline$\varepsilon / 4$ & $1.08 \mathrm{E}-02$ & $2.79 \mathrm{E}-03$ & $8.26 \mathrm{E}-04$ & 4.91E-04 & 4.65E-04 & 4.64E-04 \\
\hline rate & - & 1.95 & 1.76 & 0.75 & 0.08 & 0.00 \\
\hline$\varepsilon / 4^{2}$ & $1.08 \mathrm{E}-02$ & 2.77E-03 & 7.10E-04 & $2.21 \mathrm{E}-04$ & $1.42 \mathrm{E}-04$ & $1.36 \mathrm{E}-04$ \\
\hline rate & - & 1.97 & 1.96 & 1.68 & 0.64 & 0.07 \\
\hline$\varepsilon / 4^{3}$ & $1.08 \mathrm{E}-02$ & 2.76E-03 & $6.99 \mathrm{E}-04$ & $1.80 \mathrm{E}-04$ & $5.92 \mathrm{E}-05$ & $4.10 \mathrm{E}-05$ \\
\hline rate & - & 1.97 & 1.98 & 1.96 & 1.60 & 0.53 \\
\hline$\varepsilon / 4^{4}$ & $1.08 \mathrm{E}-02$ & $2.76 \mathrm{E}-03$ & $6.98 \mathrm{E}-04$ & $1.76 \mathrm{E}-04$ & 4.54E-05 & $1.60 \mathrm{E}-05$ \\
\hline rate & - & 1.97 & 1.99 & 1.99 & 1.95 & 1.51 \\
\hline
\end{tabular}


Table 2: Convergence of EXFD scheme (2.3) to LogKGE (1.1).

\begin{tabular}{|c|c|c|c|c|c|c|}
\hline$\left\|\tilde{e}^{\varepsilon}(1)\right\|_{l^{\infty}}$ & $\begin{aligned} h & =0.1 \\
\tau & =0.1\end{aligned}$ & $\begin{array}{l}h / 2 \\
\tau / 2\end{array}$ & $\begin{array}{l}h / 2^{2} \\
\tau / 2^{2}\end{array}$ & $\begin{array}{l}h / 2^{3} \\
\tau / 2^{3}\end{array}$ & $\begin{array}{l}h / 2^{4} \\
\tau / 2^{4}\end{array}$ & $\begin{array}{l}h / 2^{5} \\
\tau / 2^{5}\end{array}$ \\
\hline$\varepsilon=10^{-3}$ & $1.63 \mathrm{E}-03$ & $6.76 \mathrm{E}-04$ & 7.43E-04 & $7.68 \mathrm{E}-04$ & $7.75 \mathrm{E}-04$ & $7.76 \mathrm{E}-03$ \\
\hline rate & - & 1.27 & -0.14 & -0.05 & -0.01 & 0.00 \\
\hline$\varepsilon / 4$ & 1.70E-03 & 4.31E-04 & $2.14 \mathrm{E}-04$ & 2.25E-04 & $2.28 \mathrm{E}-04$ & 2.29E-04 \\
\hline rate & - & 1.98 & 1.01 & -0.08 & -0.02 & 0.00 \\
\hline$\varepsilon / 4^{2}$ & $1.71 \mathrm{E}-03$ & 4.37E-04 & 1.10E-04 & 6.58E-05 & 6.72E-05 & $6.76 \mathrm{E}-05$ \\
\hline rate & - & 1.97 & 1.99 & 0.73 & -0.03 & -0.01 \\
\hline$\varepsilon / 4^{3}$ & $1.71 \mathrm{E}-03$ & 4.37E-04 & $1.10 \mathrm{E}-04$ & $2.76 \mathrm{E}-04$ & 1.97E-05 & $1.99 \mathrm{E}-05$ \\
\hline rate & - & 1.96 & 1.99 & 2.00 & 0.48 & -0.01 \\
\hline$\varepsilon / 4^{4}$ & $1.71 \mathrm{E}-03$ & 4.37E-04 & $1.10 \mathrm{E}-04$ & $2.76 \mathrm{E}-05$ & 6.90E-06 & $5.82 \mathrm{E}-06$ \\
\hline rate & - & 1.96 & 1.99 & 2.00 & 2.00 & 0.25 \\
\hline \multirow[t]{2}{*}{$\left\|\tilde{e}^{\varepsilon}(1)\right\|_{l^{2}}$} & $h=0.1$ & $h / 2$ & $h / 2^{2}$ & $h / 2^{3}$ & $h / 2^{4}$ & $h / 2^{5}$ \\
\hline & $\tau=0.1$ & $\tau / 2$ & $\tau / 2^{2}$ & $\tau / 2^{3}$ & $\tau / 2^{4}$ & $\tau / 2^{5}$ \\
\hline$\varepsilon=10^{-3}$ & 3.73E-03 & $1.43 \mathrm{E}-03$ & $1.23 \mathrm{E}-03$ & $1.24 \mathrm{E}-03$ & $1.25 \mathrm{E}-03$ & $1.25 \mathrm{E}-03$ \\
\hline rate & - & 1.39 & 0.21 & -0.01 & -0.01 & 0.00 \\
\hline$\varepsilon / 4$ & 3.71E-03 & 9.72E-04 & $4.06 \mathrm{E}-04$ & 3.55E-04 & $3.56 \mathrm{E}-04$ & $3.56 \mathrm{E}-04$ \\
\hline rate & - & 1.93 & 1.26 & 0.19 & 0.00 & 0.00 \\
\hline$\varepsilon / 4^{2}$ & 3.72E-03 & $9.43 \mathrm{E}-04$ & 2.52E-04 & $1.15 \mathrm{E}-04$ & $1.02 \mathrm{E}-04$ & $1.02 \mathrm{E}-04$ \\
\hline rate & - & 1.98 & 1.90 & 1.13 & 0.17 & 0.00 \\
\hline$\varepsilon / 4^{3}$ & $3.72 \mathrm{E}-03$ & $9.42 \mathrm{E}-04$ & $2.38 \mathrm{E}-04$ & 6.53E-05 & 3.23E-05 & 2.93E-05 \\
\hline rate & - & 1.98 & 1.99 & 1.86 & 1.01 & 0.14 \\
\hline$\varepsilon / 4^{4}$ & 3.72E-03 & $9.43 \mathrm{E}-04$ & 2.37E-04 & 5.98E-05 & 1.69E-05 & $9.10 \mathrm{E}-06$ \\
\hline rate & - & 1.98 & 1.99 & 1.99 & 1.82 & 0.89 \\
\hline \multirow[t]{2}{*}{$\left\|\tilde{e}^{\varepsilon}(1)\right\|_{H^{1}}$} & $h=0.1$ & $h / 2$ & $h / 2^{2}$ & $h / 2^{3}$ & $h / 2^{4}$ & $h / 2^{5}$ \\
\hline & $\tau=0.1$ & $\tau / 2$ & $\tau / 2^{2}$ & $\tau / 2^{3}$ & $\tau / 2^{4}$ & $\tau / 2^{5}$ \\
\hline$\varepsilon=10^{-3}$ & 5.05E-03 & $1.92 \mathrm{E}-03$ & $1.59 \mathrm{E}-03$ & $1.59 \mathrm{E}-03$ & $1.59 \mathrm{E}-03$ & $1.59 \mathrm{E}-03$ \\
\hline rate & - & 1.39 & 0.27 & 0.01 & 0.00 & 0.00 \\
\hline$\varepsilon / 4$ & 4.93E-03 & $1.31 \mathrm{E}-03$ & $5.51 \mathrm{E}-04$ & 4.68E-04 & 4.64E-04 & 4.64E-04 \\
\hline rate & - & 1.91 & 1.25 & 0.23 & 0.01 & 0.00 \\
\hline$\varepsilon / 4^{2}$ & $4.92 \mathrm{E}-03$ & $1.25 \mathrm{E}-03$ & 3.39E-04 & $1.56 \mathrm{E}-04$ & $1.37 \mathrm{E}-04$ & $1.35 \mathrm{E}-04$ \\
\hline rate & - & 1.98 & 1.88 & 1.12 & 0.19 & 0.01 \\
\hline$\varepsilon / 4^{3}$ & 4.92E-03 & $1.24 \mathrm{E}-03$ & $3.14 \mathrm{E}-04$ & 8.76E-05 & 4.12E-05 & 3.98E-05 \\
\hline rate & - & 1.99 & 1.98 & 1.84 & 0.99 & 0.15 \\
\hline$\varepsilon / 4^{4}$ & 4.92E-03 & $1.24 \mathrm{E}-03$ & $3.11 \mathrm{E}-04$ & 7.87E-05 & 2.27E-05 & $1.25 \mathrm{E}-05$ \\
\hline rate & - & 1.99 & 1.99 & 1.98 & 1.78 & 0.86 \\
\hline$\varepsilon / 4^{5}$ & 4.92E-03 & $1.24 \mathrm{E}-03$ & $3.11 \mathrm{E}-04$ & 7.79E-05 & $1.96 \mathrm{E}-05$ & 5.92E-06 \\
\hline rate & - & 1.99 & 2.00 & 2.00 & 1.98 & 1.74 \\
\hline
\end{tabular}




\section{Acknowledgments}

The authors would like to express sincere gratitude to the reviewers for their constructive suggestions, which helped to improve this paper. This work is done when the first author visited the Department of Mathematics at the National University of Singapore in 2019-2020. The authors are very grateful to Prof. Weizhu Bao and the members of his group for valuable suggestions and comments.

This work is supported by the National Natural Science Foundation of China (Grant Nos. 11971481, 11901577), by the Natural Science Foundation of Hunan (Grants Nos. S2017JJQNJJ0764, S2020JJQNJJ1615), by the Basic Research Foundation of the National Numerical Wind Tunnel Project (No. NNW2018-ZT4A08), by the Research Fund of NUDT (Grants Nos. ZK17-03-27, ZK19-37), by the fund from Hunan Provincial Key Laboratory of Mathematical Modeling and Analysis in Engineering (Grant No. 2018MMAEZD004), by the Hunan Provincial Innovation Foundation (Grant No. CX20200010), and by Chinese Scholarship Council (Grant No. 201903170118).

\section{References}

[1] D.D. Bainov and E Minchev, Nonexistence of global solutions of the initial-boundary value problem for the nonlinear Klein-Gordon equation, J. Math. Phys. 36, 756-762 (1995).

[2] W.Z. Bao and Y.Y. Cai, Uniform error estimates of finite difference methods for the nonlinear Schrödinger equation with wave operator, SIAM J. Numer. Anal. 50, 492-521 (2012).

[3] W.Z. Bao and Y.Y. Cai, Optimal error estimates of finite difference methods for the Gross-Pitaevskii equation with angular momentum rotation, Math. Comp. 82, 99-128 (2013).

[4] W.Z. Bao, Y.Y. Cai and X.F. Zhao, A uniformly accurate multiscale time integrator pseudospectral method for the Klein-Gordon equation in the nonrelativistic limit regime, SIAM J. Numer. Anal. 52, 2488-2511 (2014).

[5] W.Z. Bao, R. Carles, C.M. Su and Q.L. Tang, Error estimates of a regularized finite difference method for the logarithmic Schrödinger equation, SIAM J. Numer. Anal. 57, 657-680 (2019).

[6] W.Z. Bao, R. Carles, C.M. Su and Q.L. Tang, Regularized numerical methods for the logarithmic Schrödinger equation, Numer. Math. 143, 461-487 (2019).

[7] W.Z. Bao, R. Carles, C.M. Su and Q.L. Tang, Error estimates of energy regularization for the logarithmic Schrödinger equation, ArXiv, 2006.05114 (2020).

[8] W.Z. Bao and X.C. Dong, Analysis and comparison of numerical methods for the Klein-Gordon, equation in the nonrelativistic limit regime, Numer. Math. 120, 189-229 (2012).

[9] W.Z. Bao, X.C. Dong and X.F. Zhao, An exponential wave integrator sine pseudospectral method for the Klein-Gordon-Zakharov system, SIAM J. Sci. Comput. 35, A2903-A2927 (2013).

[10] W.Z. Bao, Y. Feng and W.F. Yi, Long time error analysis of finite difference time domain methods for the nonlinear Klein-Gordon equation with weak nonlinearity, Commun. Computat. Phys. 26, 1307-1334 (2019).

[11] W.Z. Bao and X.F. Zhao, A uniformly accurate multiscale time integrator spectral method for the Klein-Gordon-Zakharov system in the high-plasma-frequency limit regime, J. Comput. Phys. 327, 270-293 (2016).

[12] W.Z. Bao and X.F. Zhao, A uniformly accurate (UA) multiscale time integrator Fourier pseudospectral method for the Klein-Gordon-Schrödinger equations in the nonrelativistic limit regime, Numer. Math. 135, 833-873 (2017). 
[13] W.Z. Bao and X.F. Zhao, Comparison of numerical methods for the nonlinear Klein-Gordon equation in the nonrelativistic limit regime, J. Comput. Phys. 398, 108886 (2019).

[14] J.D. Barrow and P. Parsons, Inflationary models with logarithmic potentials, Phys. Rev. D. 52, 5576 (1995).

[15] K. Bartkowski and P. Górka, One-dimensional Klein-Gordon equation with logarithmic nonlinearities, J. Phys. A 41, 355201 (2008).

[16] I. Bialynicki-Birula and J. Mycielski, Wave equations with logarithmic nonlinearities, Bull. Acad. Polon. Sci. Cl. 3, 461 (1975).

[17] I. Bialynicki-Birula and J. Mycielski, Nonlinear wave mechanics, Ann. Physics 100, 62-93 (1976).

[18] I. Bialynicki-Birula and J. Mycielski, Gaussons, solitons of the logarithmic Schrödinger equation, Phys. Scr. 20, 539 (1979).

[19] P. Brenner and W.V. Wahl, Global classical solutions of nonlinear wave equations, Math. Z. 176, 87-121 (1981).

[20] H. Buljan, A. Šiber, M. Soljačić, T. Schwartz, M. Segev and D. Christodoulides, Incoherent white light solitons in logarithmically saturable noninstantaneous nonlinear media, Phys. Rev. E 68, 036607 (2003).

[21] W.M. Cao and B.Y. Guo, Fourier collocation method for solving nonlinear Klein-Gordon equation, J. Comput. Phys. 108, 296-305 (1993).

[22] T. Cazenave and A. Haraux, Équations d'évolution avec non linéarité logarithmique, Ann. Fac. Sci. Toulouse Math. 2, 21-51 (1980).

[23] Q.S. Chang, G.B. Wang and B.L. Guo, Conservative scheme for a model of nonlinear dispersive waves and its solitary waves induced by boundary motion, J. Comput. Phys. 93, 360-375 (1991).

[24] D.B. Duncan, Sympletic finite difference approximations of the nonlinear Klein-Gordon equation, SIAM J. Numer. Anal. 34, 1742-1760 (1997).

[25] K. Enqvist and J. McDonald, Q-balls and baryogenesis in the MSSM, Phys. Lett. B 425, 309-321 (1998).

[26] E. Faou and K. Schratz, Asymptotic preserving schemes for the Klein-Gordon equation in the non-relativistic limit regime, Numer. Math. 126, 441-469 (2014).

[27] P. Górka, Logarithmic Klein-Gordon equation, Acta Phys. Pol. B 40, 59-66 (2009).

[28] J.M. Holte, Discrete Gronwall lemma and applications, in: MAA-NCS Meeting at the University of North Dakota 24, 1-7 (2009).

[29] S. Ibrahim, M. Majdoub and N. Masmoudi, Global solutions for a semilinear, two-dimensional Klein-Gordon equation with exponential-type nonlinearity, Commun. Pure Appl. Math. 59, 1639-1658 (2006).

[30] S. Jiménez and L. Vázquez, Analysis of four numerical schemes for a nonlinear Klein-Gordon equation, Appl. Mat. Comun. 35, 61-94 (1990).

[31] R. Kosecki, The unit condition and global existence for a class of nonlinear Klein-Gordon equations, J. Differ. Equ. 100, 257-268 (1992).

[32] W. Królikowski, D. Edmundson and O. Bang, Unified model for partially coherent solitons in logarithmically nonlinear media, Phys. Rev. E 61, 3122 (2000).

[33] H.W. Li, X. Zhao and Y.X. Hu, Numerical solution of the regularized logarithmic Schrödinger equation on unbounded domains, Appl. Numer. Math. 140, 91-103 (2019).

[34] A. Linde, Strings, textures, inflation and spectrum bending, Phys. Lett. B 284, 215-222 (1992).

[35] S. Machihara, The nonrelativistic limit of the nonlinear Klein-Gordon equation, Funkcial. Ekvac. 44, 243-252 (2001).

[36] V.G. Makhankov, I.L. Bogolubsky, G. Kummer and A.B. Shvachka, Interaction of relativistic 
gaussons, Phys. Scr. 23, 767 (1981).

[37] S.D. Martino, M. Falanga, C. Godano and G. Lauro, Logarithmic Schrödinger-like equation as a model for magma transport, EPL 63, 472 (2003).

[38] N. Masmoudi and K. Nakanishi, From nonlinear Klein-Gordon equation to a system of coupled nonlinear Schrödinger equations, Math. Ann. 324, 359-389 (2002).

[39] F. Natali and E.C. Jr, Orbital stability of periodic standing waves for the logarithmic Klein-Gordon equation, J. Math. Anal. Appl. 484, 123723 (2020).

[40] P.J. Pascual, S. Jiménezz and L. Vázquez, Numerical simulations of a nonlinear Klein-Gordon model. applications, in: Lecture Notes in Physics 448, 211-270, Springer (1995).

[41] G. Rosen, Dilatation covariance and exact solutions in local relativistic field theories, Phys. Rev. 183, 1186 (1969).

[42] J.J. Sakurai, Advanced Quantum Mechanics, Addison Wesley (1967).

[43] T.C. Scott, X.D. Zhang, R.B. Mann and G.J. Fee, Canonical reduction for dilatonic gravity in 3+ 1 dimensions, Phys. Rev. D 93, 084017 (2016).

[44] T.C. Scott and K.G. Zloshchastiev, Resolving the puzzle of sound propagation in liquid helium at low temperatures, J. Low. Temp. Phys. 45, 1231-1236 (2019).

[45] J.C.H. Simon and E. Taflin, The Cauchy problem for non-linear Klein-Gordon equations, Comm. Math. Phys. 152, 433-478 (1993).

[46] M.Z. Song, X. Qian, T.L. Shen and S.H. Song, Stochastic conformal schemes for damped stochastic Klein-Gordon equation with additive noise, J. Comput. Phys. Vol. 411, 109300 (2020).

[47] T.C. Wang, X.F. Zhao and J.P. Jiang, Unconditional and optimal H2-error estimates of two linear and conservative finite difference schemes for the Klein-Gordon-Schrödinger equation in high dimensions, Adv. Comput. Math. 44, 477-503 (2018).

[48] A.M. Wazwaz, Gaussian solitary wave solutions for nonlinear evolution equations with logarithmic nonlinearities, Nonlinear Dyn. Syst. Theory 83, 591-596 (2016).

[49] H. Zhang, X. Qian, J.Y. Yan and S.H. Song, Novel high-order mass-and energy-conservative Runge-Kutta integrators for the regularized logarithmic Schrödinger equation, doi: 10.13140/ RG.2.2.31979.69921 (2019).

[50] L.M. Zhang, Convergence of a conservative difference scheme for a class of Klein-GordonSchrödinger equations in one space dimension, Appl. Mat. Comun. 163, 343-355 (2005). 